\title{
Atlas of Photographs of Sections of the Frozen Cranium and Brain of the Cat (Felis Domestica).
}

\author{
By
}

\author{
R. H. Clarke, M. A., M. B. and E. E. Henderson, B. A., M. B., F. R. C. S.
}

Part I. Sagittal Sections.

(With 7 Figures in the text and Pl. 28-39.)

\section{Introduction.}

The accompanying photographs of sagittal sections of the cranium of the cat are the first instalment of a complete series of sections in three planes of the crania of the cat and monkey, which we hope eventually to complete.

These two animals are best adapted for the investigations of the structure and functions of the brain with the insulated needles and electrical currents directed by the instruments and method of topography devised by one of us (R. H. C.) for this purpose, and for the application of which a series of sections of the cranium, or photographs of them are necessary.

It appears to us that the first step for those engaged in the systematic study of the structure and functions of the brain is to endeavour to obtain an accurate knowledge of the anatomy of every tract of fibres, its course, and connections with various nerve centres or groups of cells, and as clear an idea of the elementary functions of the latter as can be attained by our present methods of electrical stimulation, observation of natural movements, and the results of injuries or disease.

We shall explain how these objects may be advanced by the method referred to and with which this work is concerned.

Anatomy. While there is still ample scope for pure histological research, especially of the cytology of the brain, with our present microscopes and methods the limits of this field appear to be in view, and beyond it the only promising means of extending our knowledge of the structure of the central nervous system is by observation of the effects of degeneration of cells and fibres. Nor does it seem likely that this method will be superseded, for however great may be the improvements of technique, it is hardly conccivable that any reagent will act on particular fibres to the exclusion of all others unless they have undergone a preliminary 
change which in some way alters their characters. Such a change is effected by the degeneration which follows injury and it is difficult to imagine any other agent which would act in such a selective way. By Marchi's well known method this principle has been applied to the investigation of superficial structures, where small lesions can be produced without injury to adjacent nervous tissues, so that the degeneration is limited to the selected areas and the tracts connected with them. But it is impossible to apply Marchi's method by ordinary operative procedures to deep regions, as the injury inevitably inflicted in exploring them occasions such wide-spread degeneration as to deprive the results of all value for the purpose in view; and so, while this method and electrical stimulation have been extensively applied to the surface of the brain and much has been learned of its structure, elementary functions, and the course of the tracts connected with it, it has been impossible to carry these investigations into regions where, after all, the most important centres and tracts are situated, and our knowledge of the latter is very imperfect. The difficulty can be overcome by the use of needles made of platino-iridium insulated to within a short distance of the points in capillary glass tubes. The needles can be introduced into the brain with very trifling injury, and can be directed to any point by the stereotaxic instrument. By attaching the proximal end of the needle to a battery, with or without an induction coil, electrolytic lesions, from the size of a pin's head to that of a pea, can be produced; or by the use of the faradic current the responses to electric stimuli can be ascertained, and both methods can be employed at the same point without moving the needle. The accidental injury caused by the passage of the needle and the resulting degeneration are slight and generally negligeable and the method therefore fulfils what is requisite for working out the anatomy of the tracts and centres of the brain in a satisfactory way.

Physiology. On the physiological side all methods of artificial excitation of the brain are still crude, but with the rapid progress of electrical science it cannot be long before they are improved. They are at present the best means available for testing the elements of localised function, they have given information of great value, and with proper precautions will continue to do so, and with the insulated needle they can be applied with precision to any desired point in the brain. Finally, whatever can be learned from clinical symptoms produced by injury of the brain is valuable in proportion to the accurate localisation and limitation of the lesion which.gives rise to them, and for this purpose artificial lesions made by this method are very suitable. These considerations show that the use of the insulated needle for the purposes indicated is of sufficient importance to the neurologist to enlist his interest in the method of its application which we shall now describe.

To direct a needle accurately to any point within the cranium, three requisites are indispensable and these are:

I. A system of intracranial topography which enables every cubic millimetre, or similar small area, of the brain to be readily identified, recorded, and referred to.

2. A stereotaxic instrument by which the needle can be mechanically directed out of sight, to any desired point in the brain.

3. A complete series of sections of the frozen cranium and brain, if possible in three planes, or photographs of them, prepared in accordance with the topographical niethod, to serve as charts or maps, to direct the instrument. 
Topography. The irregular curved surface of the cranium and its integuments affords no accurate basis for the topographical measurement of its contents and Clarke's method which we have employed, depends on rectilinear measurements from central section planes determined by prominent anatomical features. The cranium is devided into eight segments by three sections, approximately median in relation to important structures, each of which is perpendicular to the other two. They are termed sagittal, frontal, and horizontal, and the diameters of the cranium perpendicular to these planes, transverse, longitudinal and vertical respectively. The eight segments into which the cranium is divided by these sections are discribed as the right and left, frontal, temporal, occipital, and cerebellar. The three internal surfaces of each segment are those of a cube, and any point in a segment can be identified by three perpendiculars of correct length dependent from those surfaces. Measurements are made from both sides of each section plane which counts as zero.

The position of the section planes is determined by the following anatomical features:

A line is drawn from the centre of the auditory meatus (determined by a cone) to the lowest point of the lower margin of the orbit on each side; this is the basal horizontal plane. It lies almost entirely below the brain, and to secure the advantage of measurements from both sides of it, a more convenient horizontal plane is constructed above but parallel to it. The distance above it is empirical and determined by convenience and varies with the species of animal employed. In the monkey it is one quarter of the distance from the centre of the meatus to the vertex on a line perpendicular to the horizontal plane, in the cat one third, and in the hedgehog one half of this distance. The reason for this variation is that the interaural line connecting the centre of one meatus with the other, passes through the middle of the pons in the monkey, just below the surface of the pons in the cat, and still lower in the hedgehog. The three fractions given amount to about ten millimetres in each case and bring the horizontal zero plane to nearly the same relative position in all. In other species of animals other proportions may be more convenient. A frontal section carried down the line referred to, from vertex to meatus is the frontal zero plane, and the sagittal zero plane is the median sagittal section of the cranium determined by transverse measurement through corresponding points on both sides.

Some such method as this is indispensable for the precise localisation of minute areas and it has the advantage of simplifying and facilitating anatomical description by establishing a standard relation of the cranial contents to definite planes determined by prominent features like the eye and ear.

For the purpose we have in view it is better to employ a constant standard than to base the mutual relations of the structures of the brain upon variations dependent on the position in which an animal carries its head during life, a distinction which may be unsatisfactory in its application, and difficult to determine. In the primates, in brains as similar as those of the gorilla and the baboon, the relative position of structures must depend upon the view taken of the usual attitude of the animal in life; and in the kangaroo, squirrel, and even the bear, the erect position is so common that it seems arbitrary to reject their claims to it. This 
principle of topographical arrangement is also objectionable from a literary point of view. The whole vocabulary of familiar English words such as, ,above", ,below", ,before", and ,, behind" with their Latin equivalants, „superior", ,,inferior", ,anterior" and "posterior", is abolished, and in their default we are driven to such substitutes as "cephalad“, „caudad", „cerebralwards", ,caudalwise" and the like. There is something to be said for preserving the possibility of writing an intelligible description in simple language.

For the identification and record of each cubic millimetre and reference to it, the cranium of the standard animal is cut into slices or lamellae one millimetre thick, parallel to any of the three zero planes. These are numbered from it on both sides, the number of the lamella indicating the distance it lies in millimetres from the corresponding zero plane. Each lamella is divided into square millimetres by vertical and transverse lines, and in sagittal sections, which are always employed for record and reference unless otherwise stated, the vertical divisions (abscissae) are designated by numerals and the horizontal (ordinates) by letters of the alphabet; so that by stating the segment, the number and plane of the lamella, and the numeral and letter, any cubic millimetre in the brain can be immediately identified. Thus left frontal segment, sagittal lamella VI. D. Io. signifies a cubic millimetre $6 \mathrm{~mm}$ to the left of the median sagittal zero plane, $4 \mathrm{~mm}$ above the horizontal, and Io $\mathrm{mm}$ in front of the frontal, zero planes, respectively. It is advantageous to employ letters and numerals to distinguish the vertical and horizontal lines, but it is inconvenient to have to make frequent use of other letters than the earlier ones in the alphabet, as beyond these most people do not remember without counting what numbers the letters represent. It will be found to save time and trouble in working with the measurements involved in this method, to have plain markings on all instruments at distances of $5 \mathrm{~mm}$ so that they can be rapidly counted, and in square measurements to make blocks or divisions of $5 \mathrm{~mm}$ square. These can be called divisions and indicated in the same way as the square millimetres by a letter and a number, capital letters indicating divisions, and small letters millimetres. A capital letter and number will indicate the divisions $5 \mathrm{~mm}$ square and if it is desired to identify the square millimetre in that division a small letter and number can be added. The reference previously given would then read Left frontal segment, sagittal lamella VI. Div. A II mm d. 5. There is no advantage in this plan when the square millimetre is near the zero plane, but if the original reference were R. I7, i. e. vert. I8, longitud: I7, it would be easier to identify this as Divis: D. IV. mm c. 2. For purposes of record and reference it is advisable to adhere to one of the two planes for which the same distinction of letters for divisions of the vertical diameter and numbers for the horizontal is applicable, i. e. the sagittal and frontal, a different interpretation being required for the horizontal plane. If it is desired to record measurements in this plane, it is convenient to indicate both components by numbers, associating the word longitudinal or the letter $\mathrm{L}$ with one, and transverse or $\mathrm{T}$ with the other. Thus left frontal segment, horizontal lamella VI, L $4, \mathrm{~T}$, indicates a cubic millimetre $6 \mathrm{~mm}$ above the horizontal, $4 \mathrm{~mm}$ in front of the frontal, and $9 \mathrm{~mm}$ to the left of the sagittal zero planes respectively. But as it is easy to transpose the reference in one plane into any other, it is better to adhere to the sagittal plane for purposes of record and refe- 
rence. The above method of employing numbers for both components of a plane and distinguishing them by the words transverse, vertical or longitudinal, can of course, be adopted for the sagittal or frontal planes instead of referring to divisions in the way suggested, when high numbers or letters are involved. It is not necessary to make a hard and fast rule beforehand, for the use of the terms divisions, transverse, vertical, or longitudinal, at once shows which plan has been adopted, and the meaning is clear.

Stereotaxic instrument. Clarke's stereotaxic instrument is used to direct the insulated needle to any desired cubic millimetre of the brain.

It consists essentially of a rectangular brass frame rather larger than the head, over which it is lowered and to which it is finally fixed by four clamping screws in such a position that its lower border exactly corresponds to the zero horizontal plane of the cranium. It is adjusted at this level, before it is finally clamped, by resting on four supports, viz., two cones fitting the auditory meatus and two brackets resting on the lower borders of the orbits. It can be raised or lowered on these supports until, taking the cat as an example, the border is parallel with the basal plane and one third of the distance from the meatus to the vertex, and in this position it is clamped. The clamping screws are graduated so that the head is centred at the same time, i. e. the sagittal zero plane of the head and the frame coincide. On this frame, so adjusted, a sort of cage is constructed of graduated guides on which the needle carrier travels by rack and pinion movement, in three planes. The zero points of the instrument coincide with those of the cranium, and as the needle can be moved to or from any zero on the instrument, it must at the same time be moved to or from the corresponding zero planes of the head. If the distance of any point in the cranium from the three zero planes, is known, it is easy to direct the needle to it by the graduated guides. ${ }^{1}$ )

Chart sections. As we have stated, the distance of any point in the cranium from the three zero planes must he known, and to ascertain this it is necessary - to have a series of chart sections or photographs of them, preferably in three planes and prepared in accordance with the topographical method already explained. Such a series of photographs (taken by one of us, E. E. H.) is here presented and we shall now give a short account of the method of preparing them.

The cranium, which has been hardened in formalin and potassium bichromate $5 \%$ and $2 \%$ respectively, in water, is first drilled. The plane of section having been selected the cranium is drilled through, at two points at least, perpendicular to this plane, and the punctures are permanently marked along their whole course with lamp black. The punctures are thus evident in each section as black spots, and the drilling is so directed that these two points will always serve to identify the two section planes perpendicular to the plane of section. Suppose the cranium

1) The above briefly explains the principle of the stereotaxic instrument. It applies to the form which has been used by several investigators for some years. A full description of it was published in a joint paper by Horsley and Clarke in „Brain" in 1908 (7), which may be consuited for further particulars. A new instrument embodying some important improvements, has just been constructed and by the time this is in print, may be obtained from the maker, Mr. H. T. Goodwin, 35, Lennard Road, Penge, London S. E., and an account of it will shortly appear in this Journal. The new instrument will necessarily supersede the old one, and it was therefore unnecessary to give a lengthy description or illustration of the latter here. 
is to be cut sagittally, if one drill is passed through the line joining the two auditory meatûs, and a second one at the point where the frontal and horizontal zero planes intersect, in any section a line drawn from meatus to vertex, through the two points, will give the frontal zero plane, and a line perpendicular to this, through the second point, the whole length of the cranium, will indicate the horizontal zero plane. These lines are shewn in every photograph, and as soon as any structure is identified, the segment and lamella in which it lies and its distance from the other two zero planes, which comprise all the data required for directing the needle, are seen at once. This explains the object of drilling the cranium, and the principle on which the selection of points for drilling is made.

Adjustment of head. A needle a little longer than the selected diameter of the head is introduced and retained in one of the punctures perpendicular to the plane of section, and is used to adjust the head in the vice of the sawing instrument (I Fig. I and 2). The head, with this needle in position, is put for an hour in a jar of carbon dioxide snow, at the end of which time it is frozen completely through, and is then fixed in the vice of Clarke's saw. The head vice ( $\mathrm{I} 7$ ) of the saw is fixed on a carrier (25) which travels on guides (26) perpendicular to the saw and is moved by a graduated wheel (27) and screw. One complete revolution of the wheel moves the carriage $2 \mathrm{~mm}$. The wheel is graduated into roo divisions, each division being equivalent to $0,02 \mathrm{~mm}$. By this movement the head is brought under the saw and can be cut in slices of any desired thickness. In practice, however, the thickness of the slices or lamellae is determined by an extensible scale and index, the object of which will be explained later. The frozen head is firmly clamped in the vice in the required position, and its accurate adjustment is effected by movement of the vice, which is pivoted on the carriage so as to allow of rotation on vertical and transverse axes, and provided with clamping screws (29), (3I) to fix it in the correct position. The saw, which is a modified hack saw, works on a frame with vertical $(7)$ and transverse guides (2), and is raised and lowered by a screw (8). It is necessary to make certain that the plane of section of the cranium is parallel in both dimen-. sions to the plane of the saw. The needle which as already mentioned is left in one of the drill punctures, is used for this purpose. This needle is perpendicular in both dimensions to the proposed plane of section of the head. A bar (33) is fixed to the transverse guide of the saw perpendicular to the plane of the latter and projecting backwards beyond the plane of the vice. Two vertical arms (34) slide on this bar and each carries a needle (35) parallel to the bar and therefore perpendicular to the saw. This bar is raised or lowered with the saw; one of the vertical arms is slid over the bar in front of the vice $i$. e. between it and the saw, and the other arm behind it. So that the needle traversing the cranium is between the two needles (35) of the vertical arms (34) and approximately in line with them. By the rotatory adjustments of the vice the three needles are brought accurately into line and the vice is clamped in this position. Since the two needles are perpendicular to the plane of the saw, the needle traversing the cranium must also be perpendicular to the same plane in both dimensions, and therefore the planes of the saw and of the proposed section of the head, must be parallel. It is found in practise to be undesirable to cut sections of the frozen cranium less than $2 \mathrm{~mm}$ thick; if thinner they may be too fragile and liable to break. The set of the teeth 
causes the saw cut itself to destroy approximately $\mathrm{Imm}$ of cranium, each slice therefore accounts for $3 \mathrm{~mm}$ and is itself $2 \mathrm{~mm}$ thick and thus shows one surface of each of two lamellae. As each slice of the cranium represents three lamellae, one for the saw cut and two in the slice which is removed, it follows that we may arrange that the saw cut shall exactly replace millimetre $I, 2$ or 3 , and the surfaces of the two remaining lamellae constituting a slice will be exposed; that is to say, there are three variations of this plan of section available. Or we can arrange for the saw to descend between I and 2, 2 and 3 , or 3 and I, removing half of the adjacent lamellae and the resulting slice will contain one complete lamella and the halves of two others, one on each side of it. In this second plan of section the middle of each of these outer lamellae is exposed and the third complete lamella which intervenes, is not seen at all. This method also offers three variations.

Whichever of the alternative plans is selected, in order to obtain a complete series of lamellae viewed from the same aspect, it is necessary to use three heads, one for each of the variations indicated.

It need not be further explained that after deciding on the method of section to be adopted, the head must be brought into the correct position beneath the saw for the first cut, and after each section the carriage with vice and head are brought forward $3 \mathrm{~mm}$. The cranium is kept frozen by the application of fresh carbon dioxide snow. Cocoa nut shells cut of suitable depth filled with snow and applied to the cranium on both sides of the vice and held in position by sliding rings, are used for this purpose. A glass slide covered with mucilage is pressed against the surface exposed by the last cut and immediately freezes to it, thus serving to support it while the next cut is made; without this precaution there is risk of the brittle section breaking away. As soon as a slice is removed it is placed in formalin and bichromate solution in a numbered dish. It is afterwards cleaned from debris in water with a soft brush, and finally mounted in glycerine jelly between plass plates.

In a complete series of the sections exhibiting every lamella I mm thick in one plane, any macroscopic structure of the brain can be identified and its position in relation to the zero planes determined. It is an advantage to be able to compare the position in sections in all three planes, but this is not absolutely necessary. For practical work one complete series is indispensable, and as they are constantly being referred to, the mounted sections are inconvenient, they are also perishable, shrinking and becoming discoloured in the course of time. It is therefore better to have a series of photographs, such as we have here. They are, in fact, maps or charts, and their employment generally resembles the geographical method as applied to navigation, the divisions corresponding to latitude and longitude.

We have selected the method of exposing the middle of each lamella and have carried it out as accurately as we could. We must repeat, however, that as we have explained, the zero planes are determined from measurements of external anatomical features situated on an irregularly curved surface, and secondly that several crania of different sizes are employed, and have to be reduced to a common standard by proportional scales. This increases the probability of small errors which are inevitable in the process of measuring, adjusting-and sawing frozen heads. The photographs are enlarged two diametres, but in the original sections there should be exactly one millimetre between each of them. The margin of error is 
therefore small, a mistake of one quarter of a millimeter in two adjoining sections may result in these sections being a half or one and a half millimeters apart, an error equivalent to one whole lamella. For our next series of sections we shall have the advantage of an improved instrument and a drill made on a different principle, which we believe will prove more satisfactory in some respects than the present ones, and though we do not consider that in working with such materials mathematical accuracy is attainable, the errors are such as every operator must encounter in dealing with structures of such irregular shape and texture as the brain and skull, and they do not appear to us grave enough to interfere with the general utility of the method.

Extensible scales and proportional units of measurement. An allusion has already been made to these, and some further explanation is necessary, as they form an important element in the application of the whole of the method we are considering.

An obvious difficulty presents itself in applying standard measurements to the crania of animals, that since the heads of no two animals of the same species are absolutely identical in all dimensions and considerable differences are common, the measurements derived from the cranium of one animal may be quite inaccurate for that of another. This difficulty may be met, to a certain extent, by arithmetical correction. If a certain average size is adopted as a standard in each dimension for each species of animal, every head employed, after measurement in three dimensions, is compared with the standard, and the necessary corrections made in all three planes. This method is unsatisfactory, it is irksome to make constant arithmetical calculations, especially in the course of an experiment, and when introduced afterwards the error of adjustment of the needle has already been committed. The only satisfactory method is to employ extensible scales so that the units of measurement always vary with the dimensions of the different crania. If the size of the cranium or one dimension of it varies, either the number or the size of its divisions must vary in proportion. It is essential that the number of the divisions should renain the same. We have referred to the similarity of the sections or photographs to geographical maps, it is obviously as important that the number of such maps should be constant and that corresponding structures should be found in them as it is geographically that the same town should always occur in the same county.

The number of divisions being necessarily constant, any variation which is required must be in their size, hence the necessity for extensible scales by which the size of the divisions can be altered. If the principle of adjusting the size of units of measurement is introduced at all it must be adopted throughout the whole system of linear, square and cubic measurements, and applied to the stereotaxic instrument. the saw and the microtome. This object has been attained and the accompanying photographs, as well as the original sections are illustrations of the principle.

For linear measurement ( $x$ fig. 4) a spiral spring is enclosed in a tube with a slit extending longitudinally down one side of it. The spirals of the spring appear in this slit and serve as graduations for measurement. The spring can be extended or contracted by a screw. A millimeter scale is attached to the side of the tube so that 
the spirals can be made to correspond exactly to one millimeter, or to more or less as required. One of these measures is fixed to the new stereotaxic instrument in each plane. A standard cranium is constructed from the average of a number of measurements, and in the standard the measurements in each plane are in millimeters. In an experiment the first step is to measure the cranium of the selected animal; if any dimension correspondents to the same dimension in the standard the scale for that dimension is set to measure exactly millimeters; but if any dimension of the selected cranium is more or less than the standard the spirals of the spring are set to measure more or less than one millimeter in the same proportion, so there are always the same number of lamellae in each dimension but these lamellae may be one millimeter thick, or more or less. For square measurements the principle is carried out by an adjustable grating of silk threads. Four arms are arranged on a board in opposite pairs, pivoted about their centres, and studded throughout their length with either brass pins or small holes at equal distances. A fine silk thread is carried backwards and forwards between the pins or holes of corresponding arms making a series of parallel lines, those from one pair of arms crossing those from the other pair and together forming a grating of squares of equal size. The arms can be moved to any degree of obliquity by rotating on their pivots, and the more obliquely they are set the more closely the parallel threads are brought together. A millimeter scale being fixed immediately beneath each set of threads, they can be adjusted to any measurement, and then the arm is fixed by a clamping screw. The squares of the grating can thus be set to scale in both dimensions. The portion of board beneath the grating is removable, and is held in position by four screws by which it can be raised or lowered. When the grating has been adjusted a photographic plate or sensitised paper can be placed on this moveable board, and raised by the screws until it is in contact with the grating, of which a negative or positive print can be secured. A print corrected in this way is taken for each animal, one is shewn with each of the photographs in this book, made to scale and corrected in both dimensions proportionately to the measurements of the cranium represented as compared with the standard.

By thus adjusting the scales to the variations of different heads the same effect is produced as if they were all reduced to the same size. The number of lamellae in each dimension is constant, and subject to variations of symmetry, which fortunately are generally slight, identical structures in different animals of the same species have the same relative positions and measurements.

As we have stated, the cat and the monkey are the two animals best adapted for study by this method, and of these the cat is the most generally useful. It is plentiful everywhere and therefore cheap and always available, an advantage which, unfortunately, cannot be claimed for the monkey, which it is often difficult and sometimes impossible to procure. This advantage possessed by the cat, leads to another, for it enables the investigator to exercise some choice in the selection of animals, choosing those of medium size and rejecting any which vary considerably from the standard. The smaller size of the cat's brain, though for some purposes disadvantageous, is a great convenience where serial sections of the whole brain of a number of animals have to be mounted and packed in trays, the bulk of these preparations being very considerable. The cat exhibits symptoms of many 
lesions of the brain better than the monkey. The co-ordination of muscular movements in the normal monkey is so extraordinarily accurate that slight impairment of it is difficult to detect, the more so because it steadies itself by holding on to any available object with it's hands; and lastly, in 110 animal are tracts of fibres and centres of cell groups better marked and defined than in the cat. On these grounds we lave thought it advisable to begin with this animal. This series of sagittal sections will be followed by a frontal and horizontal series, and we propose to treat the monkey in the same way at a later date.

This explanation will, we hope, make the scope and purpose of the method sufficiently clear; we must, however, add few explanatory comments on some points of technical detail connected with the accompanying photographs.

Lettering and naming of structures. In deciding how far the naming of anatomical structures should be carried we have had to follow our own judgment, and we must briefly mention the grounds on which it has been formed. In the first place it is obvious that the object of these photographs is not that of the anatomical text book, and does not aim at systematic or descriptive anatomy, and is still less concerned with comparative morphology, and even if these objects were desirable the photographs are too small to illustrate much detail. Our object is to furnish the operator with the data which will enable him to direct the needle by means of the stereotaxic instrument, and it is only necessary to name structures which will be of use to him, for this purpose. Even within this limit there are points which it is not easy to decide. For instance it might be convenient to the operator if fi- gures were introduced to show the average extent of structures like certain nuclei which occupy adjoining parts of several lamellae, whether they were actually visible in the photographs, or not. But it would be objectionable to burden photographs of the cat's cranium, when enlarged only two diameters, with so many figures as this would involve, especially, if it were not always evident what structures were indicated, and we considered that it would be better not to attempt this object.

For purely topographical purposes, chart sections and measurements are likely to supersede anatomical landmarks and relations based upon them, for we believe that central planes and geometrical measurements from them, are much more accurate guides to localisation, especially of deep structures, than the superficial conformation of convolutions and fissures which are liable to considerable variation. But, on the other hand, while descriptive anatomy is generally beyond the scope of this work we have thought it desirable to introduce a certain amount of description and a few supplementary illustrations for the benefit of the practical worker, whether veteran or novice. The latter will probably want some description of the cat's brain and there are not many available, nor are they to be found on every lookshelf, and anyone may find that, in addition to the photographs of sections he sometimes requires to refer to the preserved brain or to dissections of it, to plan the course of the needle, etc. As suitable preparations may not be at hand, photographs or drawings of them will be of assistance.

The photographs explain themselves, and taken witl the sections, illustrate the cerebrum sufficiently without any detailed description. As far as we know, a complete classical description of the cat's brain has not yet been published, and 
there are still debateable questions of morphology which have not been finally settled. As a rule we have followed recognised descriptions, and in the few instances indebted to Flatan and Jacobsohn (4), here are mainly for that of the dog, the latter of which has been adopted by Trendelenberg (6). Elliot Smith (I) has dealt with the subject chiefly from the stand-point of parative morphology. We do not venture to criticise any of his conclusions from that standpoint, but it is not ours, which is simply topographical, and we are not sure that descriptive anatomy and comparative morphology can always be brought into line. It appears to us that in some cases, either homology or description must be sacrificed, and those who are mainly concerned with the practical application of topographical anatomy, will prefer to accept the fact that apparent similarity is not necessarily homology, and retain some terms which have been generally adopted, even though open to objection, rather than venture on all that a strictly scientific terminology would involve. For example, the Sylvian fissure in man and the monkey is not homologous with the fissure that goes by that name in the cat ([Elliot Smith (I)]; but the term has been generally adopted and in use for a considerable period and several of the gyri and sulci of the hemisphere are called by the name Sylvian or a compound of it. It would be very inconvenient to rename them all and to stamp the unfortunate furrow with imposture by calling it pseudo-Sylvian; and if pseudoSylvian why not ecto-pseudo-Sylvian, supra-pseudo-Sylvian, etc.? We think it a lesser evil to accept established names admitting of course that the nomenclature is morphologically incorrect. Another instance of conflict between the objects of scientific morphology on the one hand and practical utility on the other may be found in the fissures of the vermis cerebelli; morphologically these are of the greatest importance, but in the cat, for descriptive purposes, the secondary fissure at any rate is of no value. The secondary fissure of Elliot Smith separates the pyramid from the uvula, it is developed early and found in many species, and he reckons it next in importance to the primary fissure. But in the adult cat the lobules forming the nodule, uvula, and pyramid, are generally offshoots of a common stem of white matter, and there is often a very insignificant fissure between the uvula and pyramid, making it difficult to say whether the secondary fissure is defective or represented by a, usually deep, fissure between the pyramid and sigmoid lobe, a question more likely to puzzle the expert than enlighten the novice.

\section{The brain of the cat.}

The general conformation and the disposition of the cerebral sulci and gyri can be apprehended most easily by studying the drawings and photographs in which we have indicated the descriptive terms usually adopted. A few additional words of comment and explanation are necessary.

In the drawings of the standard head we have indicated the intra-lateral sulcus as dividing the intra-lateral gyrus throughout its length. We have no records of the point but our impression is that in the majority of cats there is no sign of an intra-lateral sulcus. In most of the remainder it appears in some rudimentary form such as a slight groove, or a fissure extending only a short distance forwards, and not to the full extent, as shown in the drawing; occasionally it is fully developed. 
The gyrus which bounds the crucial sulcus is often called the sigmoid gyrus, but it is also called the crucial gyrus, and we prefer that name. In the first place it is not sigmoid but $U$ shaped, and secondly the sigmoid bend of the vermis cere-

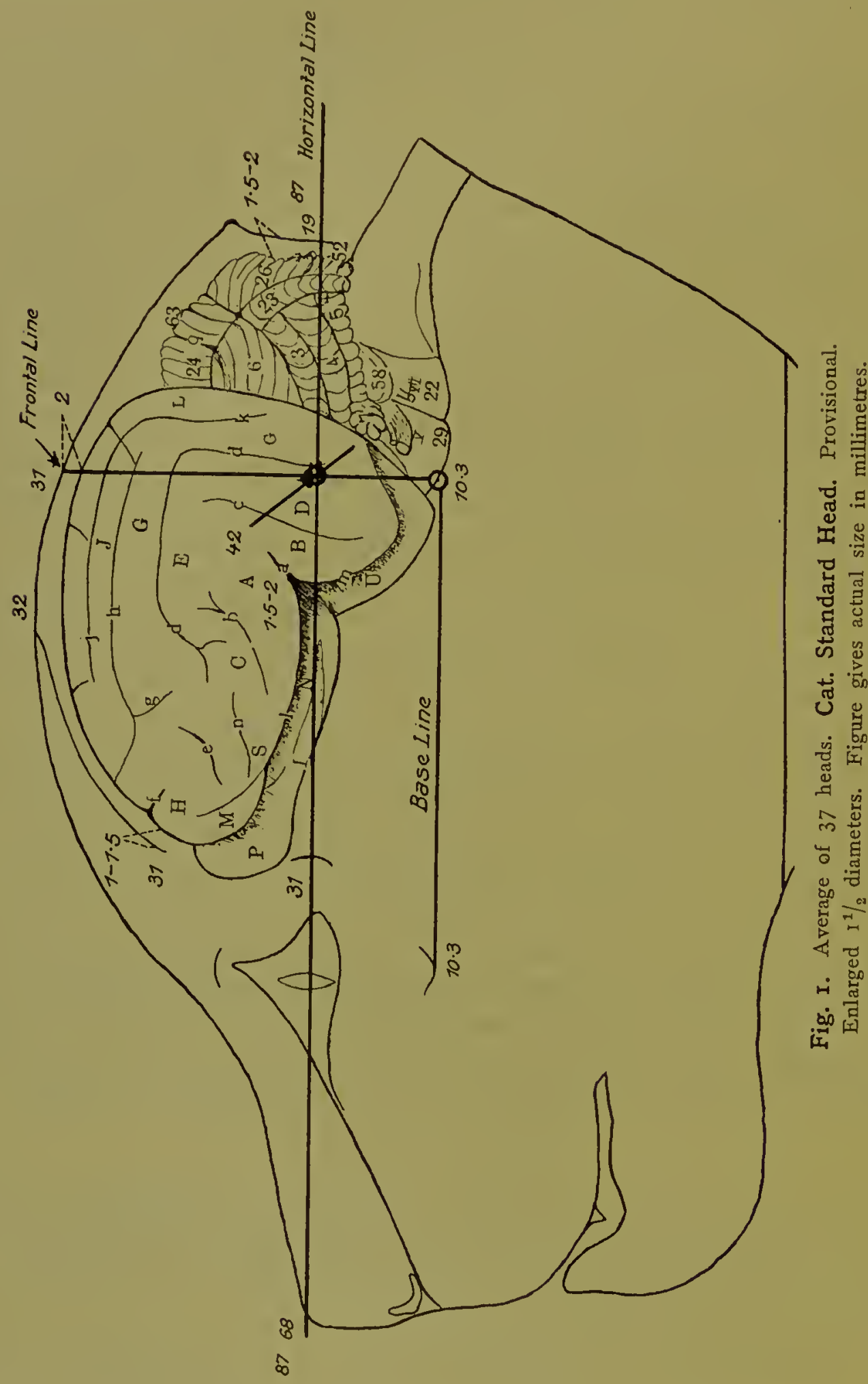

belli forms an almost perfect letter $S$ and is a most characteristic feature of the cat's cerebellum, and so obvious that if a child were given a cat's cerebellum and told to find the letter S, he could hardly fail to do so; we therefore think that the term sigmoid should be reserved for a structure which it exactly describes. Finally the 

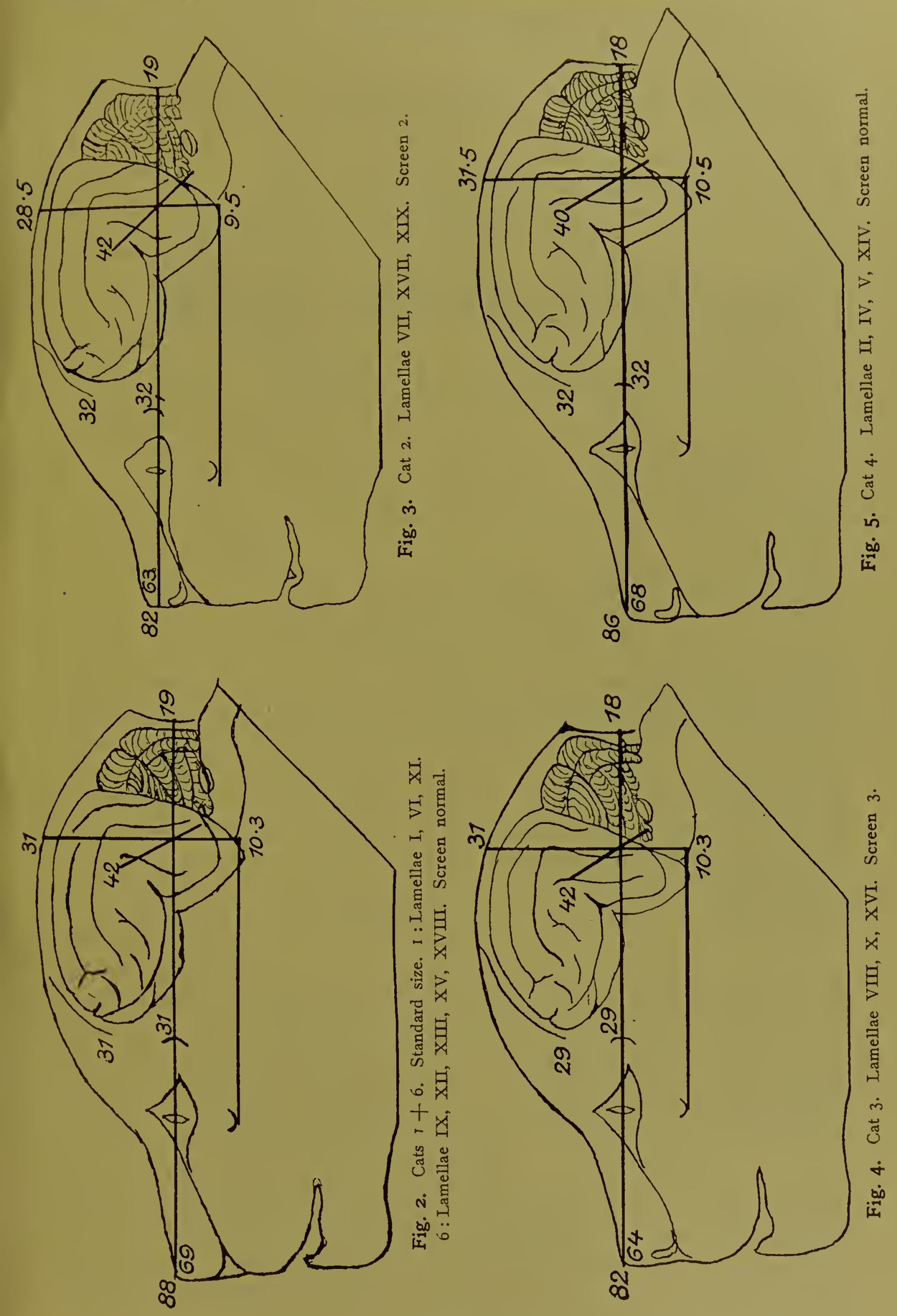
crucial sulcus is such an important landmark that it is fitting that it should give its name to the gyrus that bounds it.

Of the deeper structures shown in the photographs the most notable features are the extensive hippocampus, terminating both below and above in a cornu am-

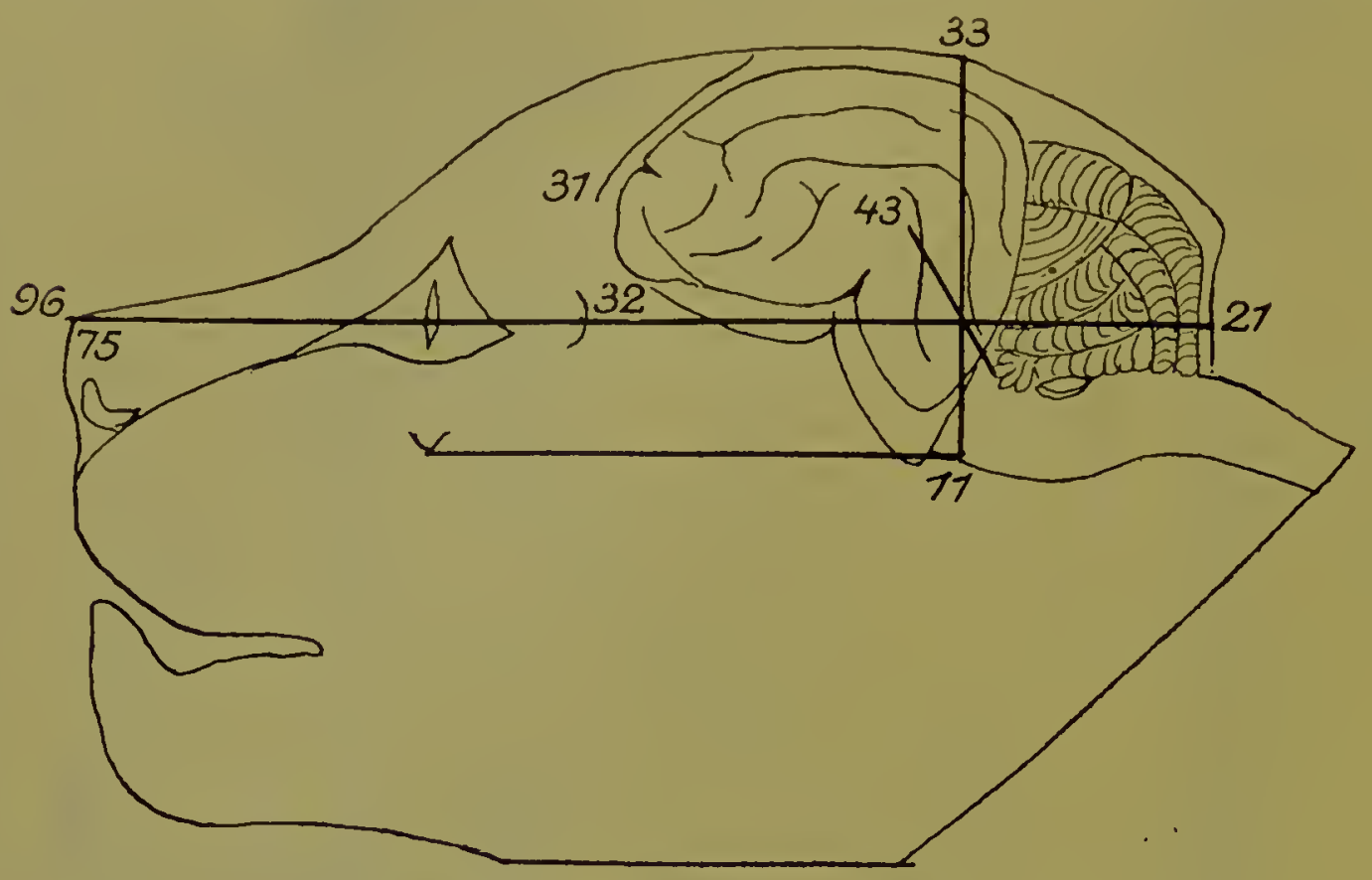

Fig. 6. Cat 5 Lamellae III. Screen 5.

monis; the large size of the posterior colliculus and the corpora geniculata, and of these latter the internal geniculates are more widely separated, and therefore external to the external geniculates or what correspond to them in man, an arrange-

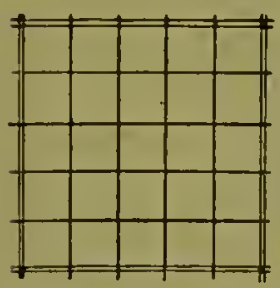

Screen normal

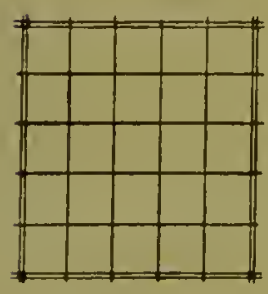

Screen 3

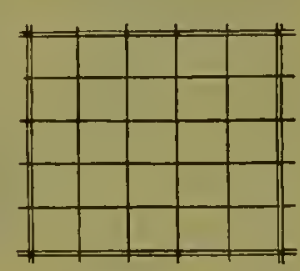

Screen 2

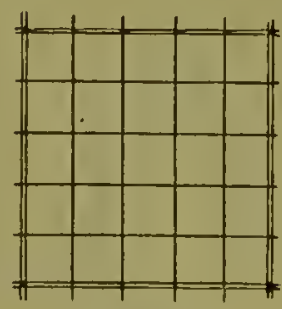

Screen 5

Fig. 7 . ment which may give rise to error and furnishes an illustration of the disadvantage of selecting such an unstable character as an essential distinction.

The cerebellum. This will require a more particular description, as owing to its complicated arrangement and the liability to variation of some of its anatomical features in animals of the same species there is some risk of misapprehension, and the published descriptions are not altogether satisfactory.

Viewed from above, the cat's cerebellum resembles a moth, the body being represented by the vermis and the outspread wings by the lateral lobes. The vermis consists of a centre of white matter in the form of two cones united at their apices like an hour glass disposed transversely to the median sagittal plane, so that its expanded extremities form the centres of the lateral lobes. It gives off numerous branches which radiate from its transverse axis and break up into twigs covered with grey matter forming lobules and these again are divided superficially into transverse ridges or folia by grooves. 
On section in the median sagittal plane the appearance is that of a tree, indicated by the old term ,arbor vitae". The narrow elongated form of the vermis and the division throughout of its cortex into annular ridges by transverse grooves, gives it some resemblance, as the name implies, to a worm, encircling the white matter, beneath which its head and tail almost meet to form the roof of the fourth ventricle. The outline of the vermis on median sagittal section is almost quadrilateral, presenting anterior, superior, posterior, and inferior surfaces, which are on the whole, the most convenient divisions for description and reference, but the lobules and folia have been grouped and named and may be described as follows:

Beginning with the head of the vermis, this is situated in front of the middle of the inferior surface, and is formed by one lobule, the lingula, superficially divided into two or sometimes three, folia. Proceeding forwards and then upwards the rounded corner of the inferior and anterior surfaces is formed by the lobus centralis, extending from the lingula to the top of the posterior colliculus which is in contact with it. The lobus centralis occupies the foremost part of the inferior and lower part of the anterior surfaces and is divided superficially into about six folia. Extending from the top of the colliculus to the primary fissure, a deep division found about the middle of the superior surface of the vermis, is a large lobe called the culmen, this includes the upper half of the anterior and front half of the superior surfaces and is divided into ten or twelve folia. The primary fissure is of horseshoe shape in outline, and descends on both sides to the superior peduncles separating the culmen from the anterior folia of the pennate lobes which are inserted into the middle peduncles. Immediately behind the primary fissure are two folia which are notable because they are generally continuous with two of the anterior pennate folia of the lateral lobes to be described presently. The position of these folia immediately behind the primary fissure, is that of the declive or lobus clivi of anatomists. Behind these two folia the vermis begins to bend to the left to form the first of three more or less horizontal limbs which. together make a complete letter S. This is a very prominent anatomical feature and is conveniently described as the sigmoid lobe. It occupies the hind part of the superior and the greater part of the posterior surfaces of the vermis. Below it the rest of the posterior surface is formed by a more or less vertical but irregular limb of about four folia, the pyramid, terminating below in a prominent angle or spur which rests on the obex of the fourth venticle. It is generally separated from the sigmoid lobe by a deep fissure and on median sagittal section has a well marked pyramidal shape with its apex terminating anteriorly in the central white matter. The inferior surface is completed by two lobules, the nodulus behind the lingula from which it is separated by a slight interval, constuting the tail of the worm, and like the lingula consisting of one lobule superficially divided into two or three folia, and the uvula, completing the inferior surface and extending from the nodule to the pyramid, änd generally presenting three or four superficial folia. The divisions of the nodule, uvula, and pyramid are often irregular, indeed all the divisions of the vermis are subject to variation, but the above is the most usual arrangement. It will be seen that according to this description the four surfaces of the vermis are constituted as follows: Anterior surface, lobus centralis and culmen. Superior surface, culmen, lobus clivi i. e. the two folia continuous with anterior pennate 
folia, and the first part of the sigmoid lobe. Posterior surface, sigmoid lobe and pyramid, inferior surface, lobus centralis, lingula, nodule, and uvula. This surface forms the roof of the fourth ventricle.

The lateral lobes are placed symmetrically on either side of the vermis, and the same description applies to both. Each lobe is of triangular form, slightly curved forwards, the base being attached to the vermis, and presenting two surfaces, an upper and a lower, surrounded by a steeply sloping border, which descends outwards from roof to floor. The upper surface is formed by the pennate lobe, consisting of two series of folia, anterior and posterior, arranged something like the barbs of a feather obliquely on both sides of a median fissure, which extends from near the middle of the base towards the apex of the lateral lobe. The floor is formed by the three peduncles, superior, middle, and inferior which connect the cerebellum with the tectum, pons, and medulla respectively. The border extends from the posterior surface of the vermis behind to the culmen in front. Posteriorly it begins with a vertical column of six or seven folia divided by transverse grooves, lying alongside the posterior surface of the vermis, but not parallel to it, as its lower extremity inclines towards its fellow of the opposite side to form an incomplete V. It is known as the paramedian or paramesial lobe. From the outer border of the paramesial lobe two tiers of vertical folia, like rows of incisor teeth one above the other, encircle the lateral lobe. The upper is called the dorsal and the lower the ventral paraflocculus. The latter is less regular and sometimes incomplete; at its apex, i. e. at the tip of the lateral lobe, one or two of its folia project beyond the rest as a round nodule called the petrosal lobule. Just short of the culmen in front, the ventral paraflocculus is continuous with the dorsal and the folia connecting them are disposed in an even curve; with a similar but smaller curve about 3 folia are reflected from the anterior part of the ventral paraflocculus beneath the petrosal lobule to form the flocculus, and behind this is a bulbous enlargement, which receives the roots of the eighth nerve, the tuberculum acusticum. A large and strongly marked band of fibres, the trapezium, descends immediately beneath the tuberculum acusticum to encircle the medulla, the 7 th nerve emerging from its upper part, and in front of the trapezium below the flocculus, is the pons, formed by the expansion of the middle peduncle. The upper part of the pons is completely covered by a broad longitudinal band of fibres, the spinal root of the fifth nerve.

The centre of the vermis is composed of white matter which expands and extends on each side into the lateral lobes. The central portion is traversed in front by numerous bands of transverse fibres, the cerebellar commissure, and behind this there are some important groups of cells, the nuclei of the vermis. Similar groups of cells in the lateral extensions of white matter form the nuclei of the lateral lobes. In man and in the monkey the central nuclei consist of two pairs, the roof nucleus and nucleus globosus, arranged symmetrically on each side of the middle line, the former near the centre and the latter external and posterior to it. The lateral nuclei also consist of two on each side, the nucleus dentatus farthest out and the embolus on its inner side. This nomenclature is hardly applicable to the cat which has more than four nuclei on each side, sometimes as many as six or eight, and it is not very profitable to try and force them into an artificial nomenclature which does not precisely describe them. It seems to us better to divide 
them into two groups, viz: the central group or nuclei of the vermis, consisting of the roof and accessory nuclei, limited on each side by sagittal lamella IV, and the lateral group or nuclei of the lateral lobe, comprising the dentate and accessory nuclei. This division is probably physiological as well as anatomical. For if we compare the cerebellum of the bird with that of the monkey, the chief characteristic of the former is the large size of the vermis and the roof nuclei. Each of the latter in some birds consists of three well marked divisions bulging into the fourth ventricle and connected by extensive tracts with a vestibular apparatus, every part of which, nuclei, tracts and semicircular canals gives evidence of exceptional organization. On the other hand the dentate nuclei are minute, and the lateral lobes rudimentary, appearing to be little more than excrescences of the vermis. It seems probable that this arrangement is connected with the constant need of adjustment of equilibrum in flight; and we may contrast these structures and functions with those of the monkey, which has remarkably well developed lateral lobes, and the largest dentate and embolus proportionally, we have seen in any animal, and large and well developed as they are they are connected like the two hemispheres of the brain to form one structure. These nuclei are connected through the superior peduncle with the red nucleus, nucleus of the third nerve, and extensively with the lateral nucleus of the thalamus. Such a mechanism suggests the visual direction of muscular movement, and the monkey possesses this faculty in an extraordinary degree. Arboreal monkeys spend much of their existence leaping from bough to bough in situations where any mistake would be fatal, but no one ever sees them miss a catch; and another illustration of this faculty has been noticed by Sherrington, if a monkey is offered a grain of rice or corn on the outstretched palm, without the slightest apparent effort, and almost without looking, the animal takes it with one finger and thumb, and never touches the hand. In these instances and possibly as a general rule we may suppose that the vermis and its nuclei are chiefly concerned in co-ordinating muscular and vestibular impressions for the adjustment of equilibrium, while the lateral lobes and their nuclei convey information derived from muscular impressions to centres associated with movements of the eyes. We cannot pursue this point further, but it is in accordance with recent experimental observations of the cerebellar nuclei, and we think the division of the central and lateral groups rests on quite a different footing from the characters which distinguish the individual nuclei from one another, these are artificial and variable and not founded, so far as our present information goes, on constant or characteristic distinctions of structure or function.

Before concluding these introductory remarks we desire to express our grateful thanks to Dr. Gordon Holmes and Dr. Gordon Lane for kind assistance. To the former we are particulary indebted for valuable advice and for kindly revising our work. This was all carried out in Professor Vaughan Harley's Laboratory, and from him and Dr. Goodbody, Assistant Professor, we have always received every assistance and facility.

We need only add that we are sensible of the ambitious character of the task we have undertaken and of its inevitable defects, some of which might have been avoided by professed anatomists. But no one else, as far as we know, has the necessary instruments, and we saw no prospect of the work being done unless we began it. 


\section{References.}

1. "On the homologies of the cerebral sulci." Elliott Smith, G. Journal of Anatomy and Physiology 1902. p. 309.

2. "The primary subdivision of the Mammalian cerebellum." Loc-cit. 1902. p. 383 .

3. "The Mammalian cerebellum". "Journal of comparative neurology." V. 1895.

4. Flatau, E. und Jacobsohn, L. "Handbuch der Auatomie und vergleichenden Anatomie des Zentralnervensystems der Sãugetiere." I. Makroskopischer Teil. S. 264. Karger, Berlin 1899.

5. Langley, J. N. „The structure of the Dog's Brain." Journal of Physiology IV. 1884. p. 248.

6. Trendelenberg, W. "Sonderabdruck aus Handbuch der physiologischen Methodik." S. 88.

7. Horsley and Clarke. „The Structure and Functions of the cerebellum examined by a new method." Brain Part CXXI. Vol. XXXI. 1908.

\section{Explanation of table 28.}

Fig. I. Clarkes saw for cutting sections of cranium and brain frozen with $\mathrm{CO}_{2}$ snow.

I and 2. Male and female blades of transverse guide of saw.

3. Frame of saw.

4. Tension screw.

5. Blade of saw.

6, 6. Blade guides to prevent deflection of saw by hard structures e. $g$ teeth.

7, 7. Vertical guides of saw.

8. Screw for raising and lowering saw.

9. Grip held by catch in which screw (8) works.

Io. Spring to regulate pressure of saw.

II. Stays.

I 2. Levelling screws.

I3. Foot plate.

I4, I5. Upper jaws of head vice.

16. Screw for adjusting upper jaws of vice.

17. One of lower jaws of head vice.

18. Screw for adjusting lower jaws of vice.

19, 19. Screw guides for jaws of vice.

20. Frame of vice.

21. Clamping screw of vice (right).

22. Vertical jaws of vice.

23. Toggle joint for closing vertical jaws.

24. Screw for closing vertical jaws.

25. Travelling stage carrying vice.

26. Guide (right) for travelling stage. The stage is moved on two guides by a graduated wheel and screw.

27. Graduated wheel. One revolution moves stage and vice $2 \mathrm{~mm}$.

28. Pivot of horizontal rotatory adjustment of vice.

29. Clamp for fixing horizontal rotatory adjustment.

30. Pivot of vertical rotatory adjustment of vice.

31. Clamp for fixing vertical rotatory adjustment.

32. Plane indicator for determining adjustment of vice. It consists of a horizontal arm perpendicular to the plane of the saw carrying two vertical arms one in front of the vice, the other behind it. The vertical arms carry needles perpendicular to the plane of the saw. The whole is raised and lowered with the saw. The head is traversed by a needle perpendicular to the plane of the proposed section. When the head is in the vice the three needles are brought accurately into line and then the plane of 
the proposed section must be parallcl to the plane of the saw. The vice is clamped in this position.

33. Horizontal arm of indicator perpendicular to plane of saw.

34,34 . Vertical arms of indicator parallel to plane of saw.

35,35 . Needles of indicator perpendicular to plane of saw.

36,36 . Moreable rings to hold cocoa nut shells of suitable depth filled with $\mathrm{CO}_{2}$ snow and pressed over the head on both sides of the vice when required to freeze it.

37. Adjustable scale.

38. Index of adjustable scale.

39. Clamp for sliding ring.

Fig. 2. Saw with head of cat in vice for sagittal sections. A needle is passed through two cones from the centre of one meatus auditorius to the other. By the rotatory adjustments of the vice this needle is brought accurately into line with the two ncedles of the plane indicator and the vice is fixed by the clamping srcews in this position. As the transfixing needle is perpendicular to the sagittal plane of the cranium and the needles of the indicator perpendicular to the plane of the saw, the planes of the cranium and saw are parallel.

Fig. 3. Clarke's Drill with head of cat in position for drilling interaural line from the centre of one meatus to the other.

Fig. 4. Extensible scales (Clarke's).

I. Scale used in stereotaxic instrument. The scale consists of a spiral spring enclosed in a tube with a longitudinal opening exposing the spirals of. the spring $(a)$. The spring is extended or contracted by the rod $(b)$ which is fixed by the screw (c). At $(d)$ is a spiral joint by which the spring can be moved in the tube so as to bring any particular spiral of the spring exactly opposite the zero mark on the instrument, this spiral then counts as zero.

2. A smaller form of the same instrument, used with the saw. The spirals are painted red and white in alternate threes, because the vice of the saw is moved forwards three divisions for each section, as explained in the introduction. In the figure the extensible scale in shown in the guage which is used to set the spirals to scale.

3. Guage for setting spirals to scale. The extensible scale is held in position by screws $g, h$. The bed in which the scale rests can be moved freely by the stud $(j)$ and fixed any where by a screw underneath not seen in the figure. Two moveable indices $e$ and $f$ point both ways $\mathrm{i}$. e. to the spirals and the divisions of the millimetre scale. It will be seen that in the figure they show that 2 I divisions of the spiral scale measure $23 \mathrm{~mm}$. The screw $k$ fixes the indices. When set correctly the extensible scale is fixed by the screw $(c)$ and can than be removed from the guage and placed wherever it is required.

For the explanation of tables $29-38$ see table 39 . 


Journal f. Psychol. u. Neurol. X VIII. Ergänzungsheft.

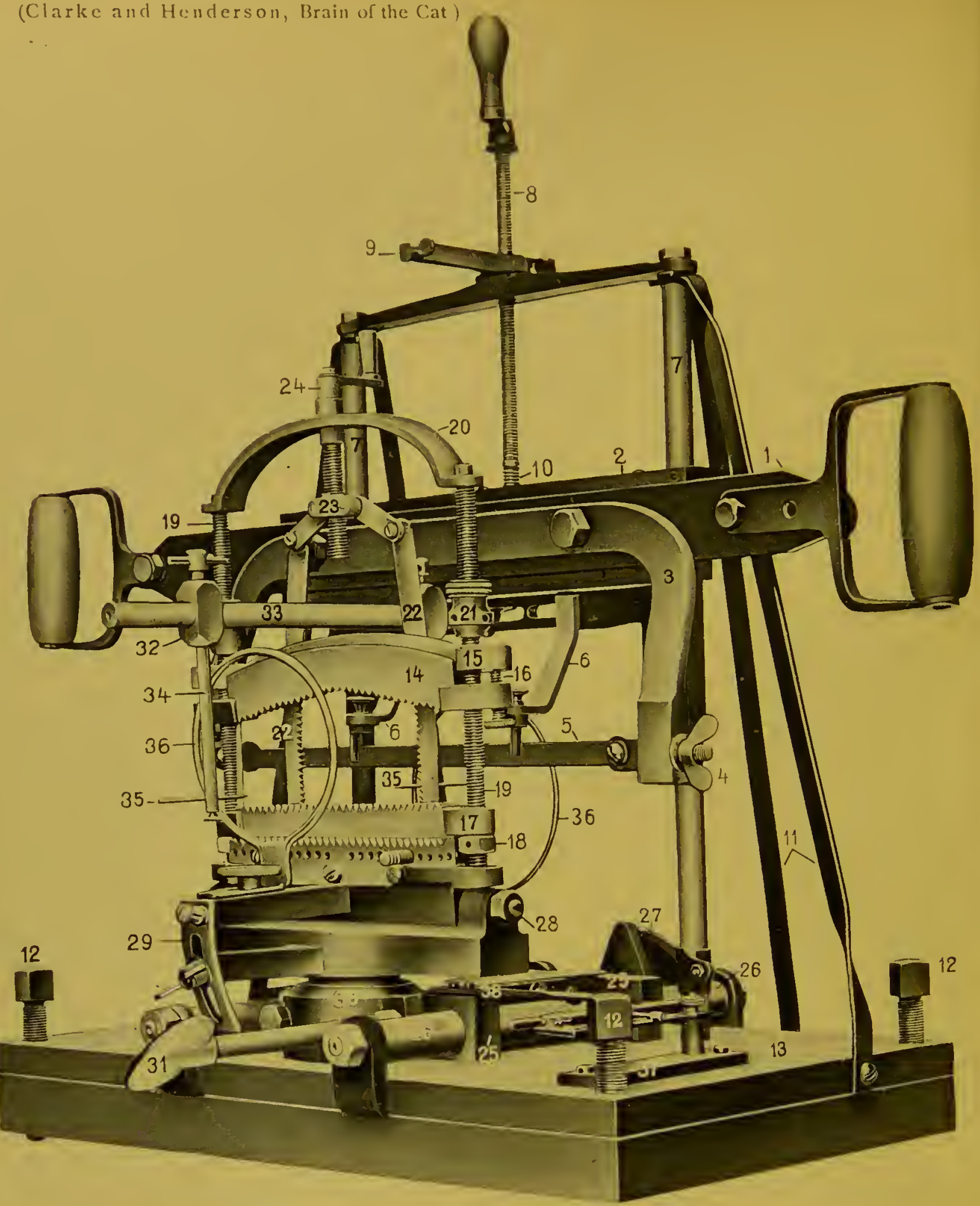

Fig. 1

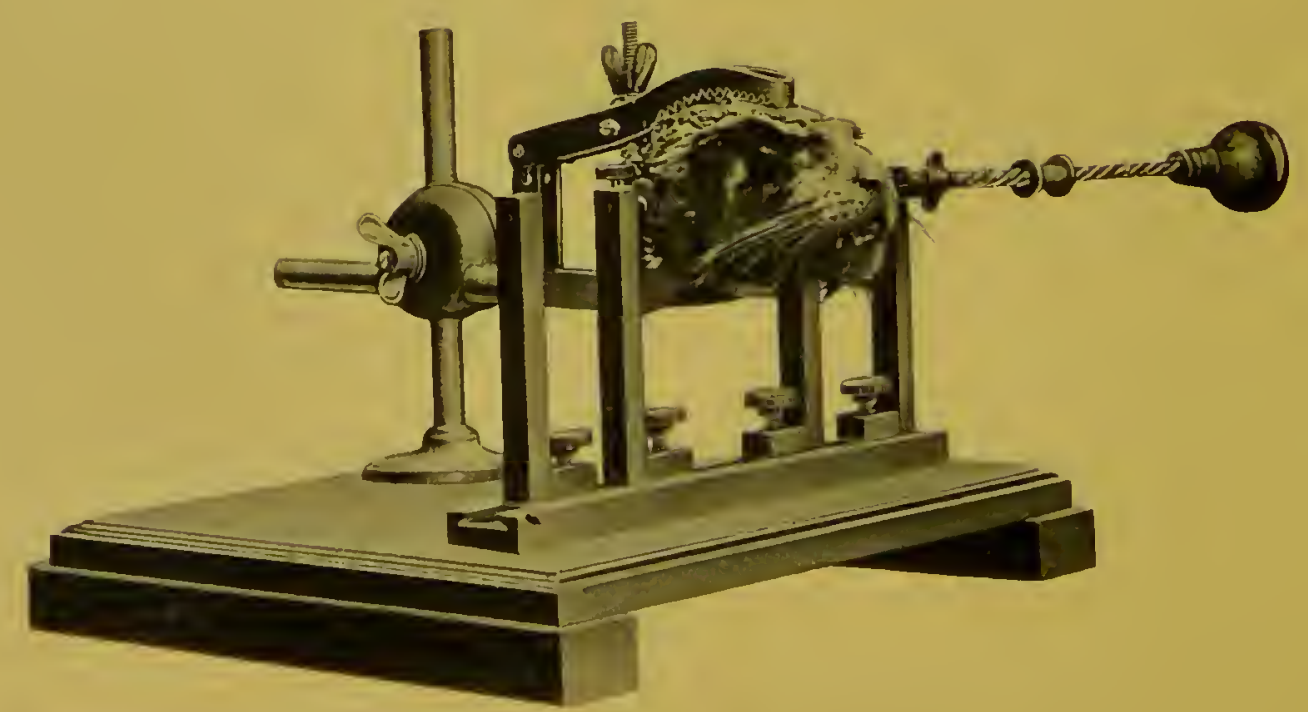




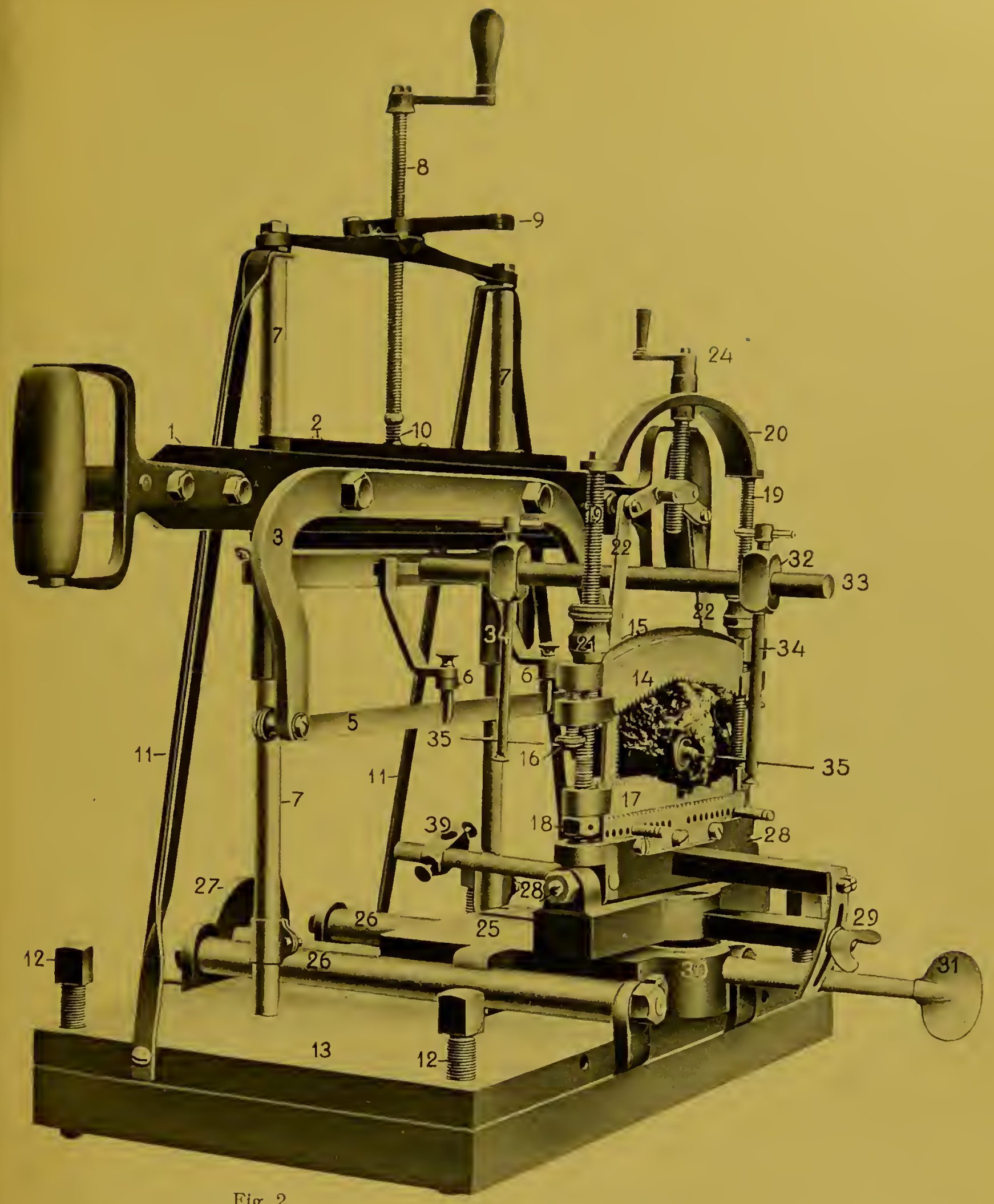

Fig. 2

1.
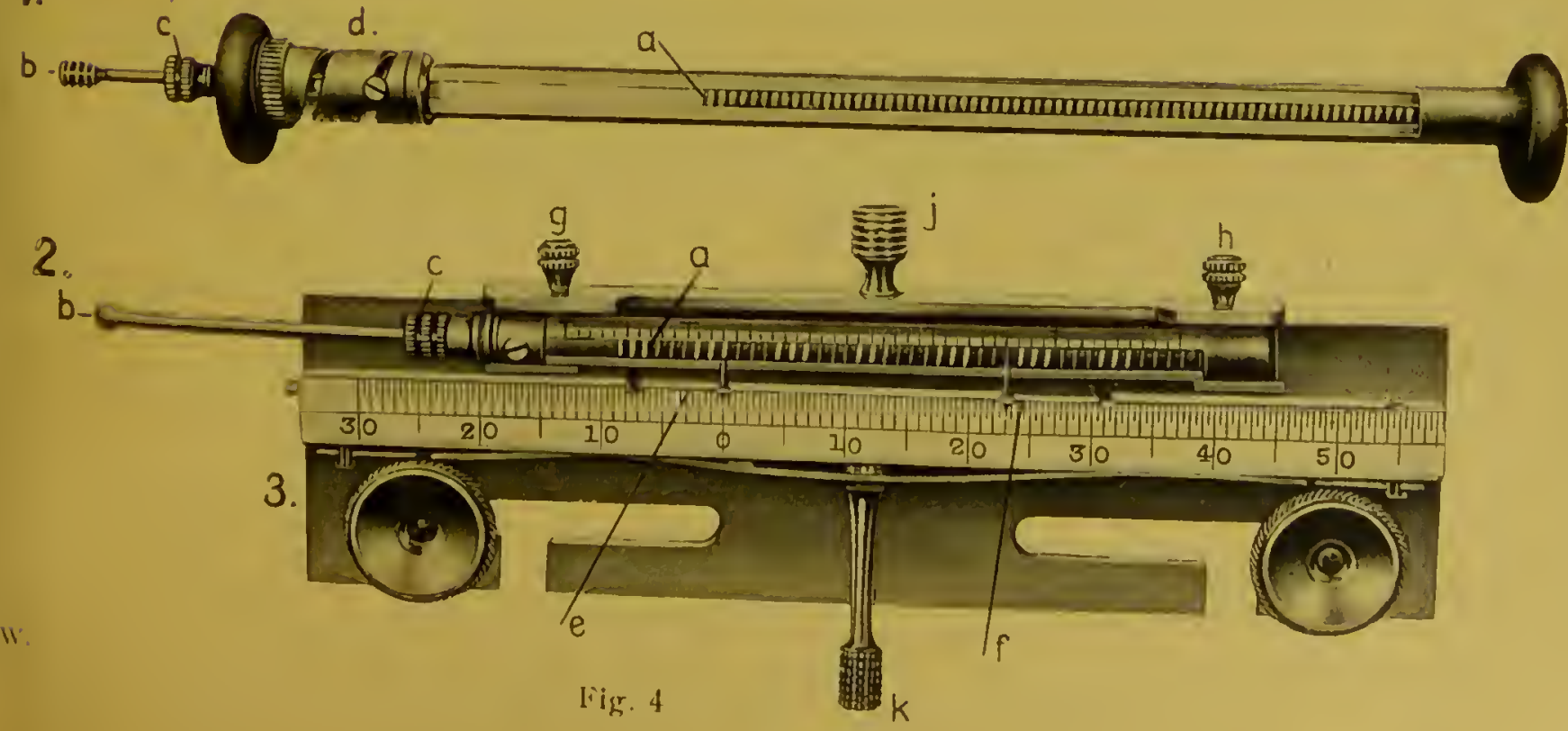

Journal f. Psychol. u. Neurol. XVIII. Ergänzungsheft. (Clarke and Henderson, Brain of Cat.)
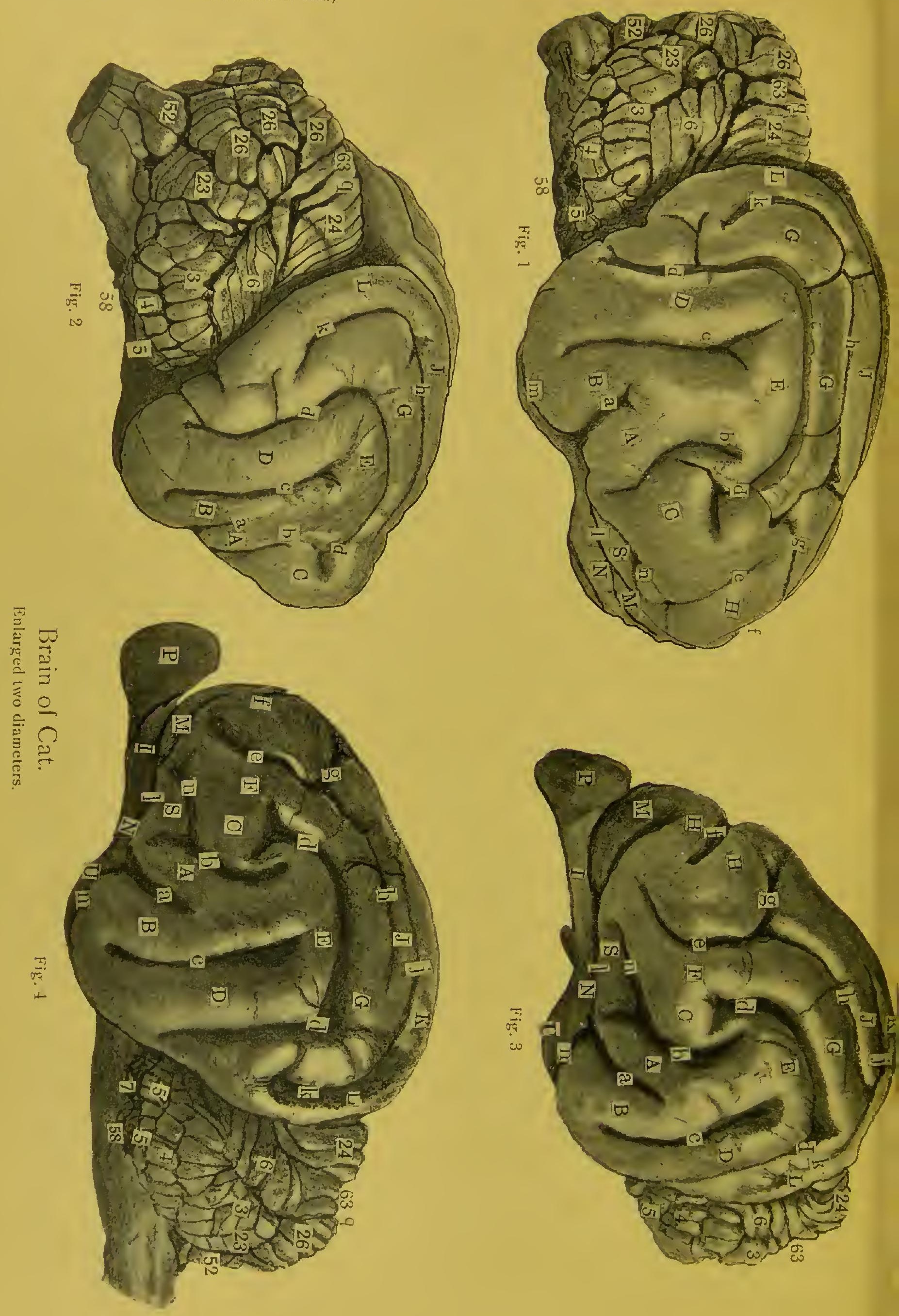


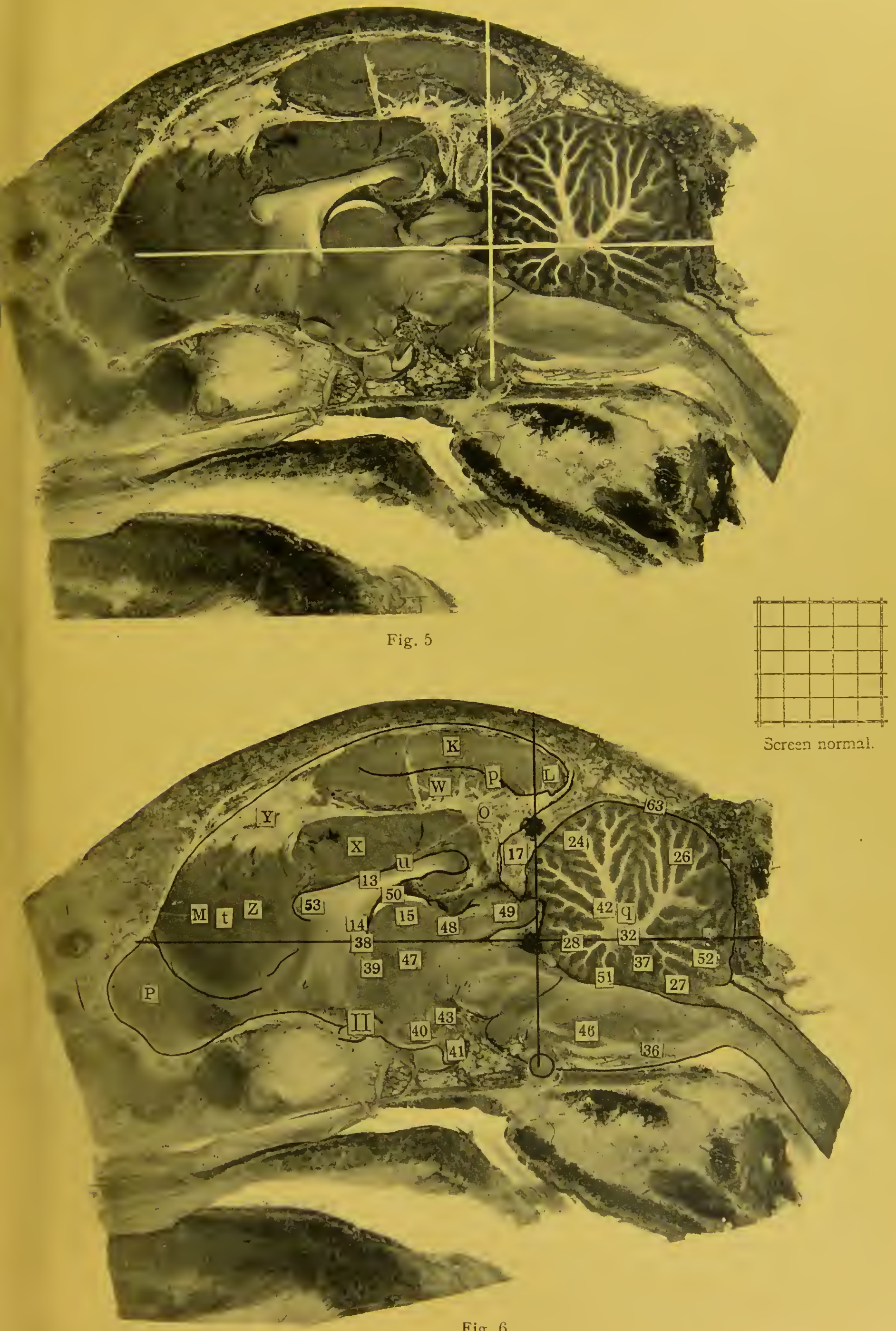

Fig. 6

Left. Sagittal Lamella 1. Cat. 1.

Enlarged two diameters. Screen normal. 

ournal f. Psychol. u. Neurol. $x / 11$. Ergänzungshett.

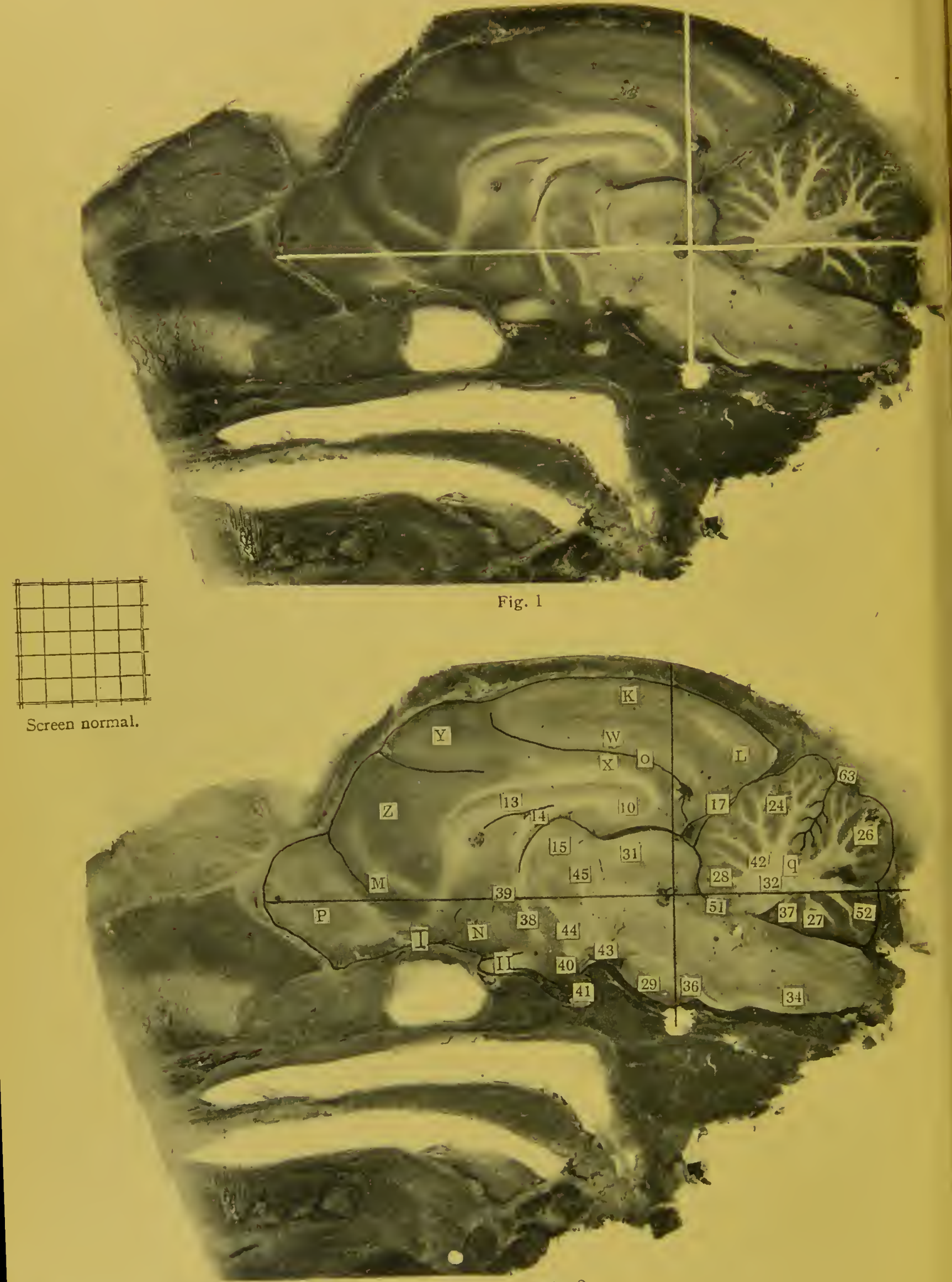




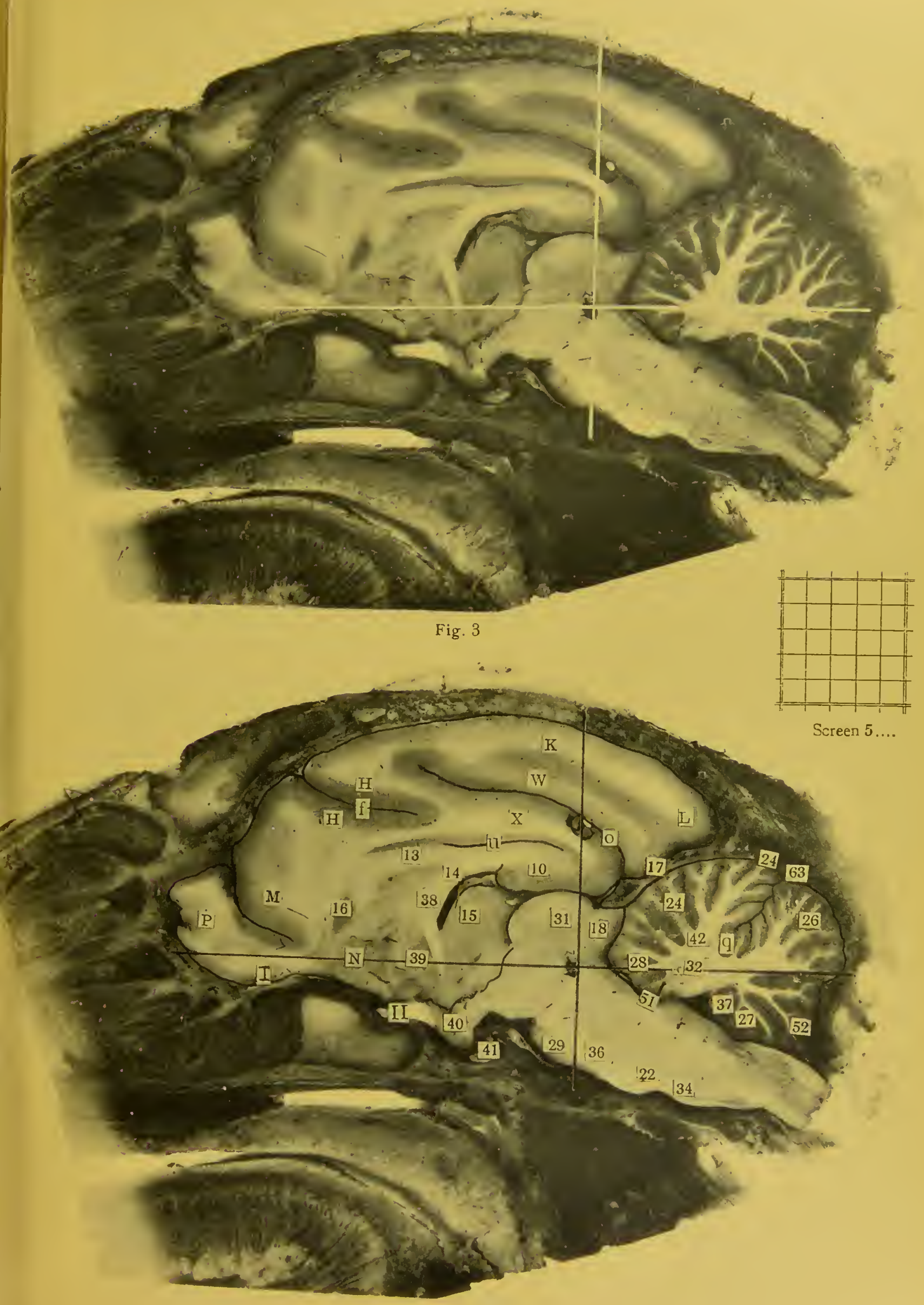

Fig. 4

Left. Sagittal Lamella 3. Cat 5. Enlarged two diameters. Screen 5. 


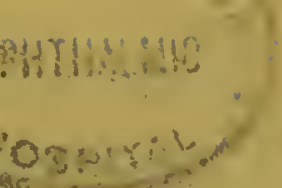


200

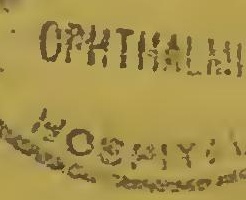


Journal f. Psychol. u. Neurol. XVIII. Ergänzungsheft.

(Clarke and Itenderson, Brain of Cat.)
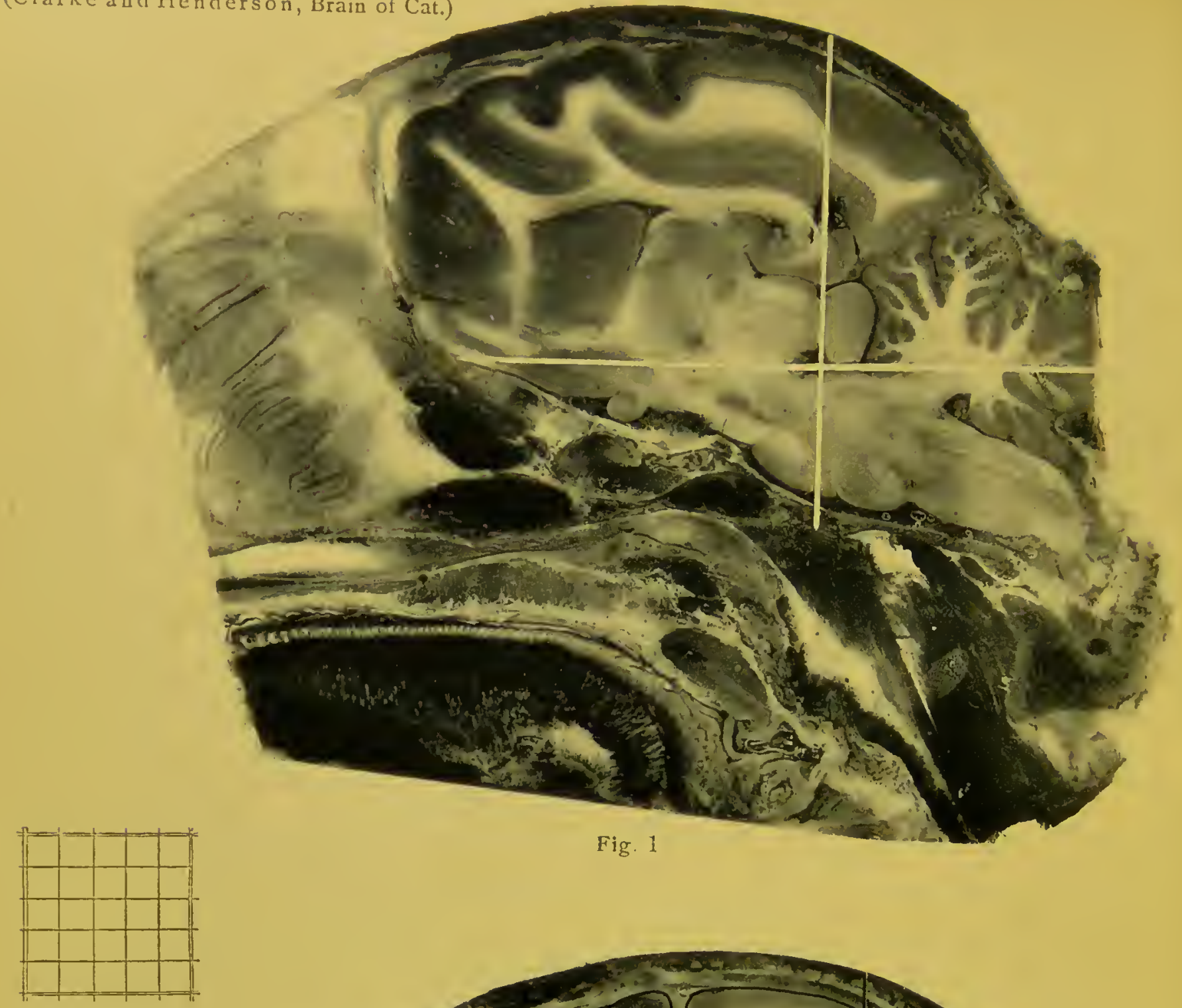

Screen normal.

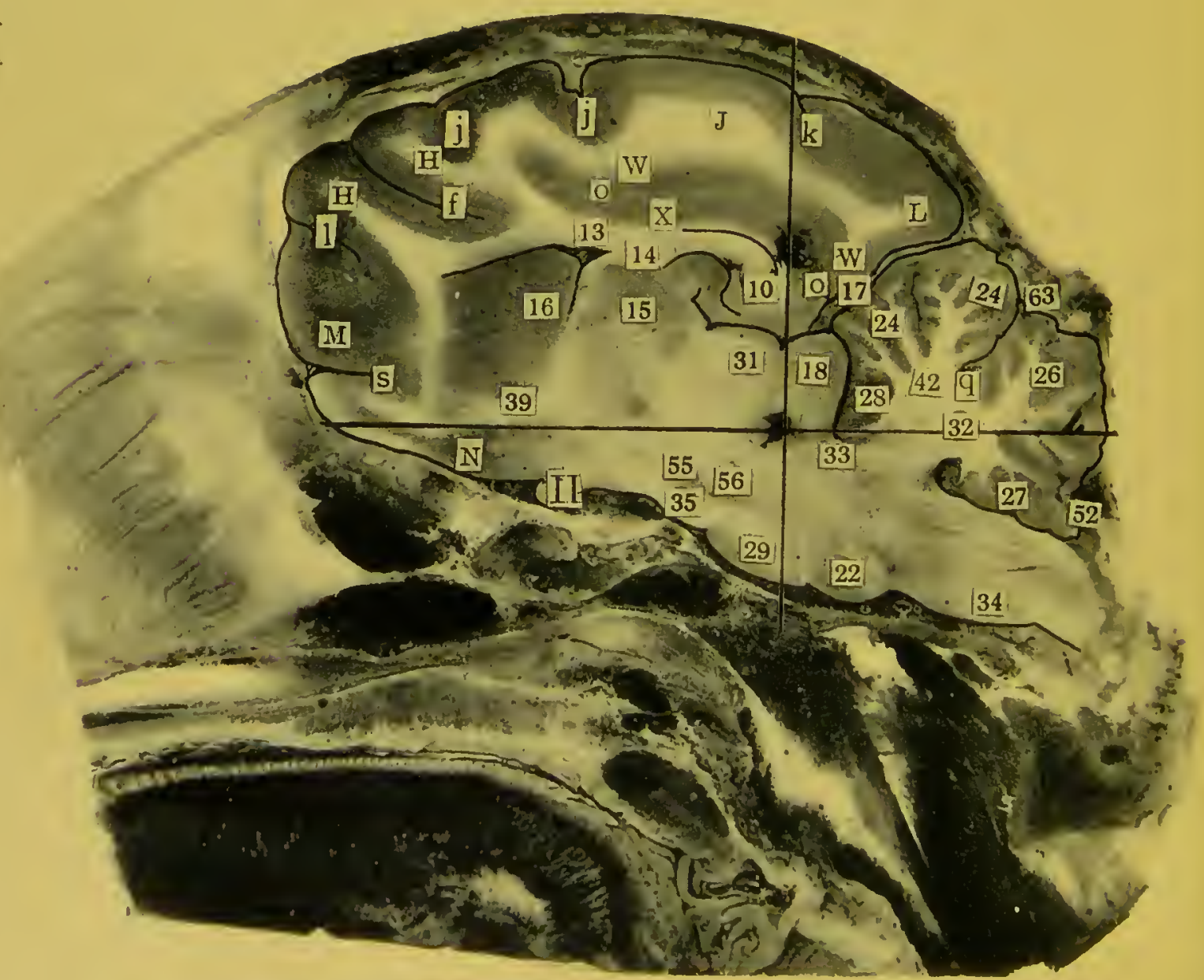

Fig. 2

Left. Sagittal Lamella 4. Cat 4.

Enlarged two diameters. Screen normal. 


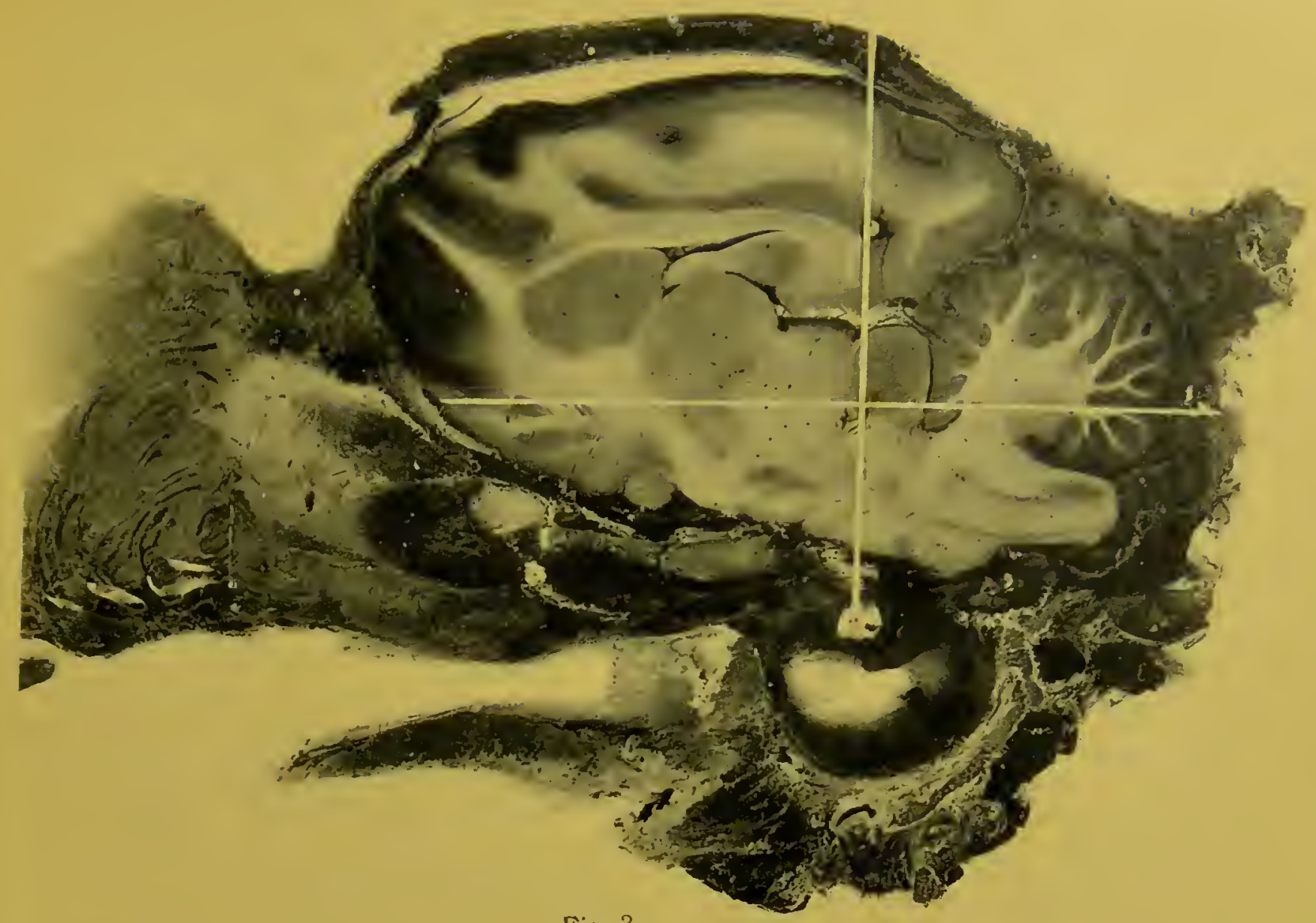

Fig. 3

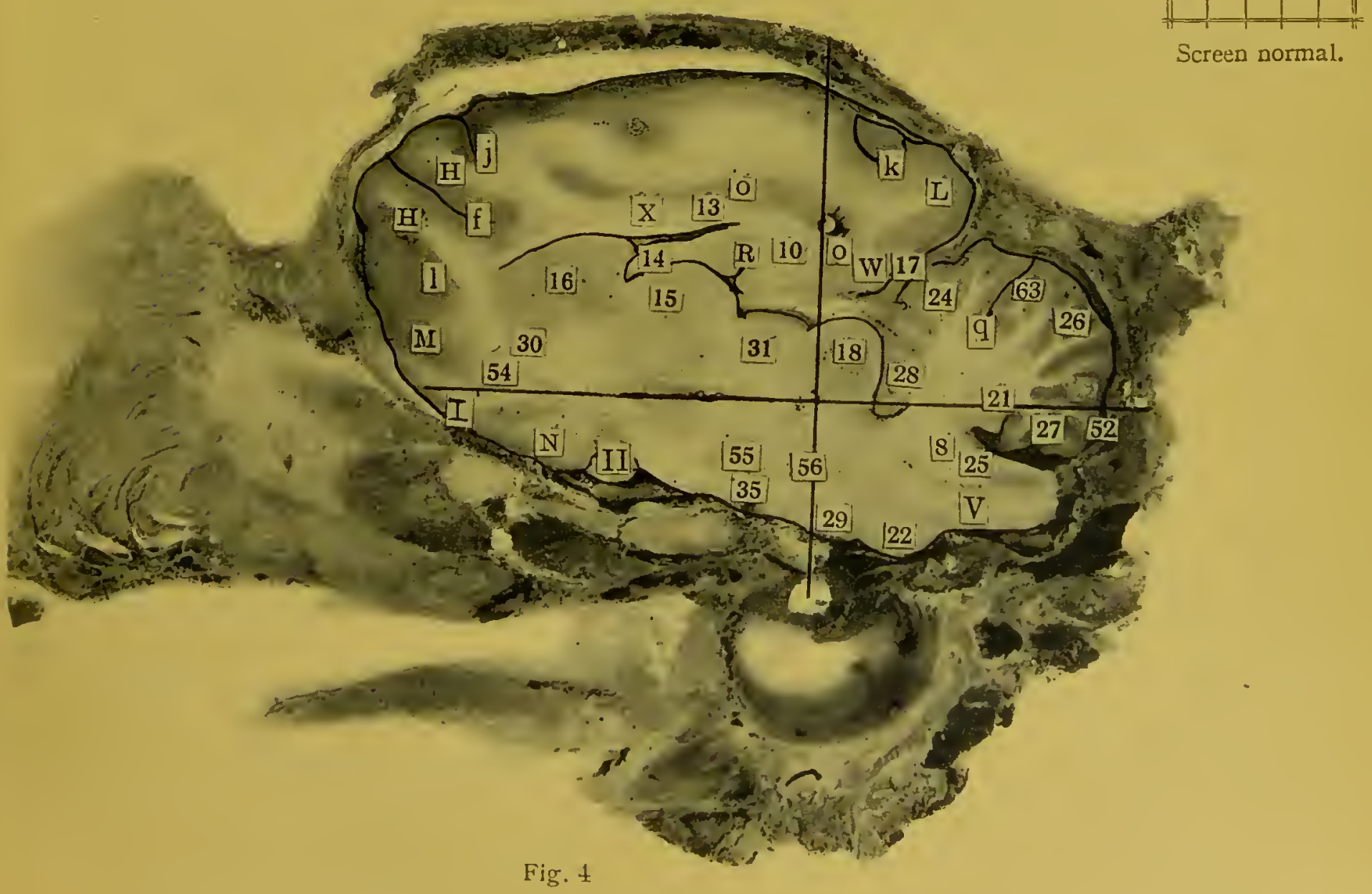

Left. Sagittal Lamella 5. Cat 4.

Enlarged two diameters. Screen normal. 
$3)$ 

Journal f. Psychol. u. Neurol. XVIII. Ergänzungsheft.

(Clarke and Henderson, Brain of Cat.)

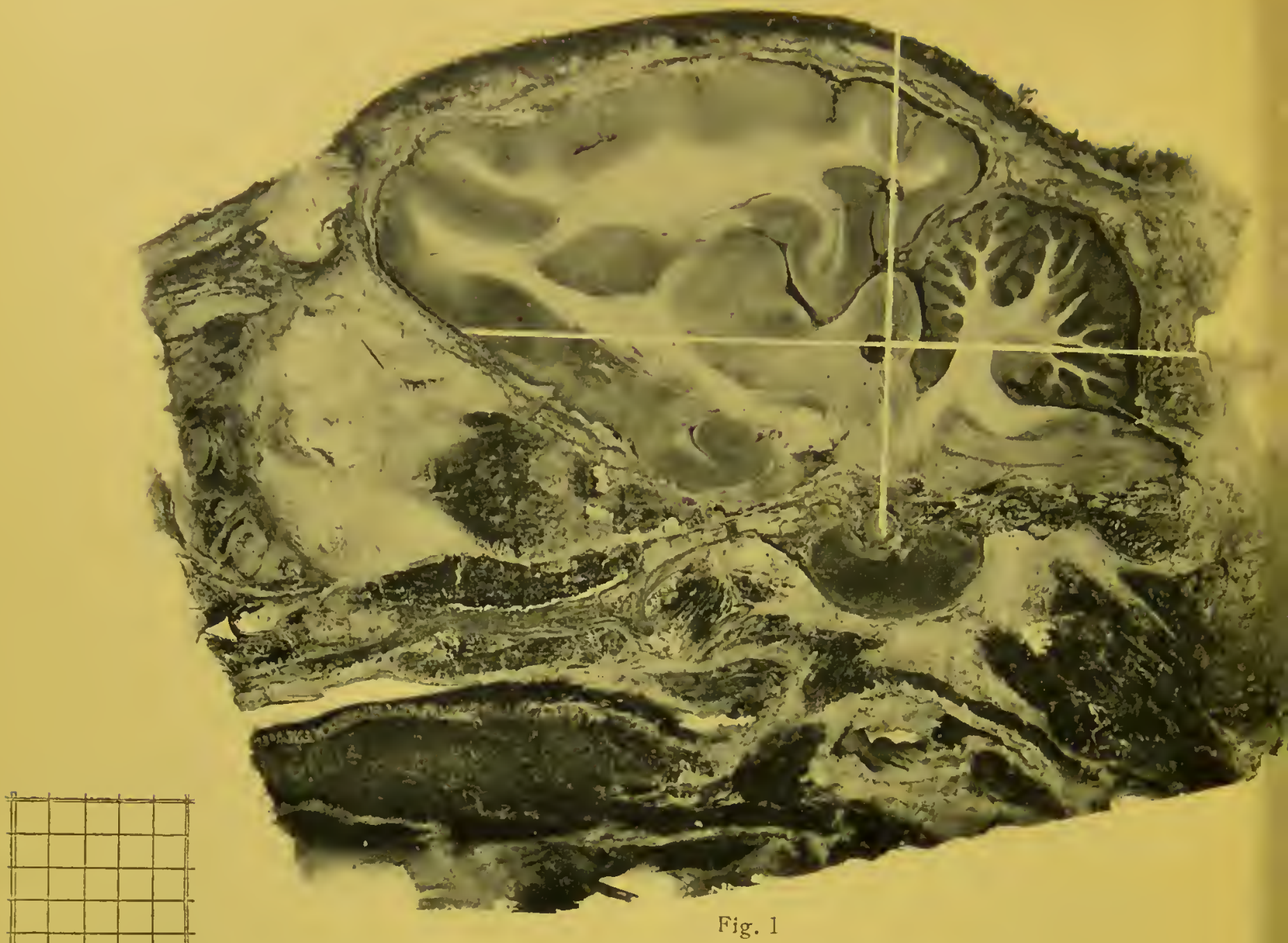

Screen normal.
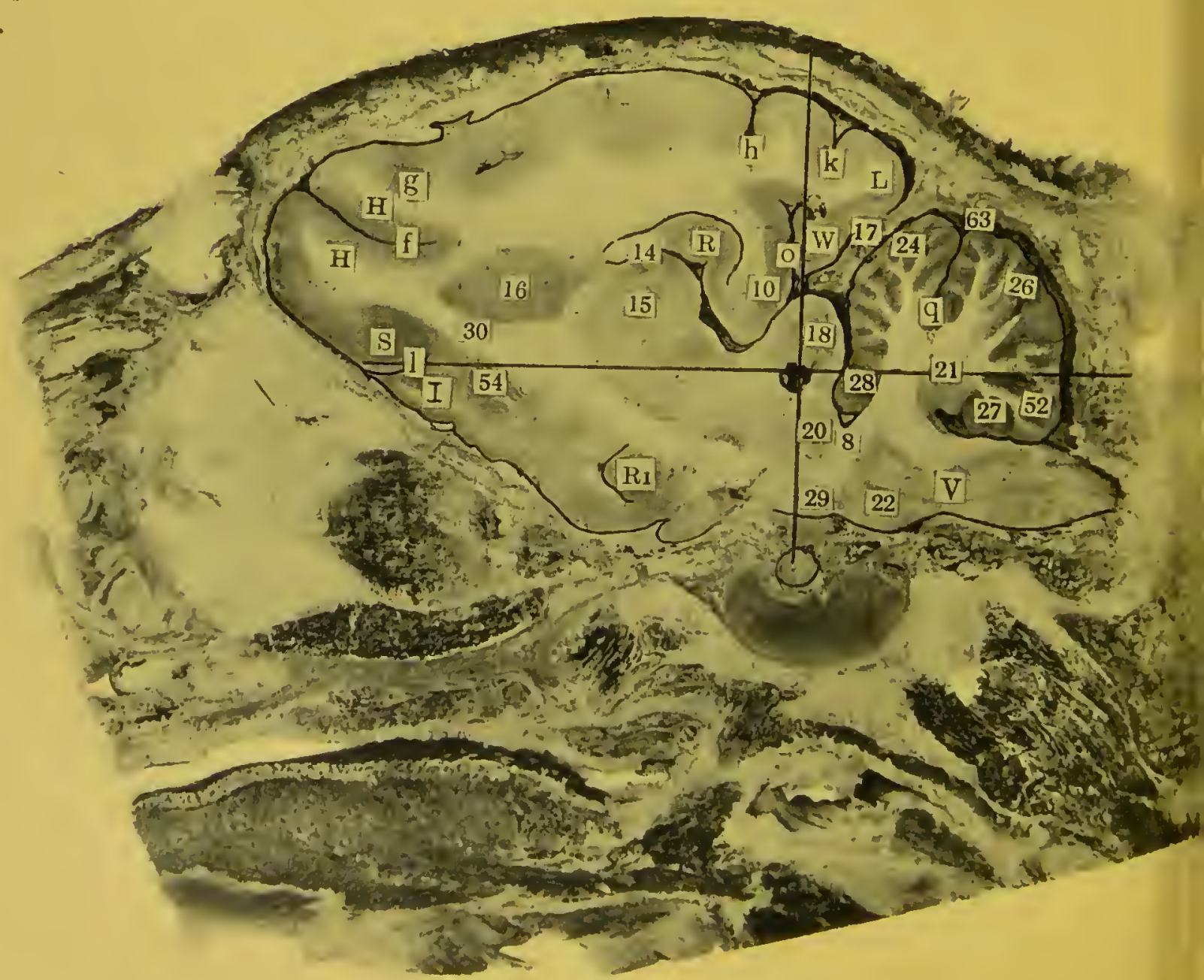

Lichtdruck von Albert Frisch, Berlin IV.

Fig. 2

Left. Sagittal Lamella 6. Cat 1.

Enlarged two diameters. Screen normal. 



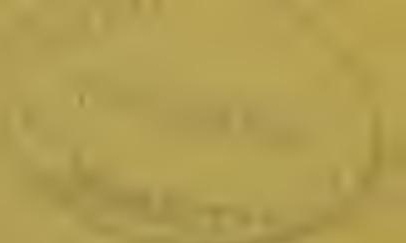

$-$ 
Journal f. Psychol. u. Neurol. XVIII. Ergänzungsheft.

(Clarke and Henderson, Brain of Cat.)
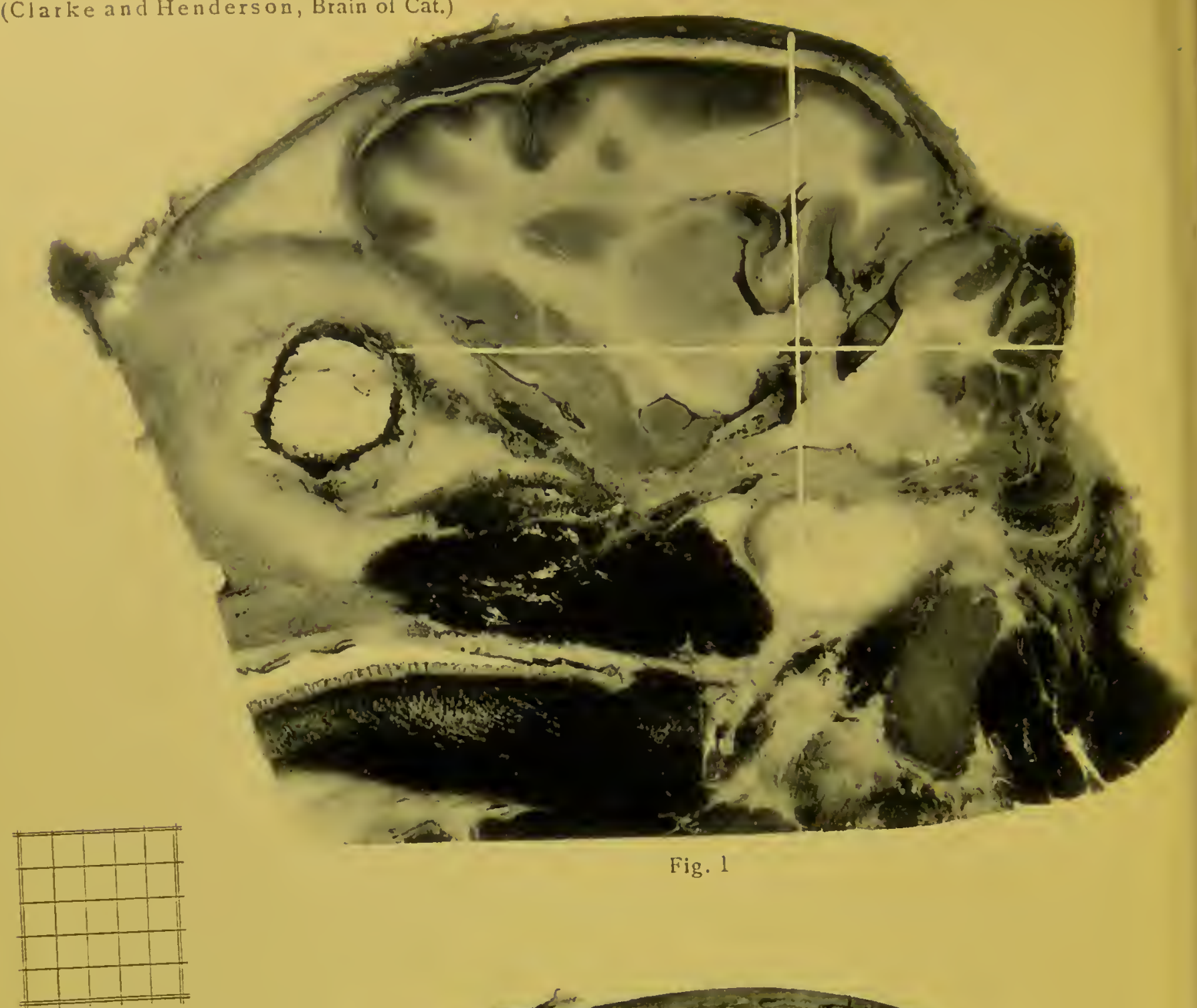

Fig. 1

Screen $3 \ldots$

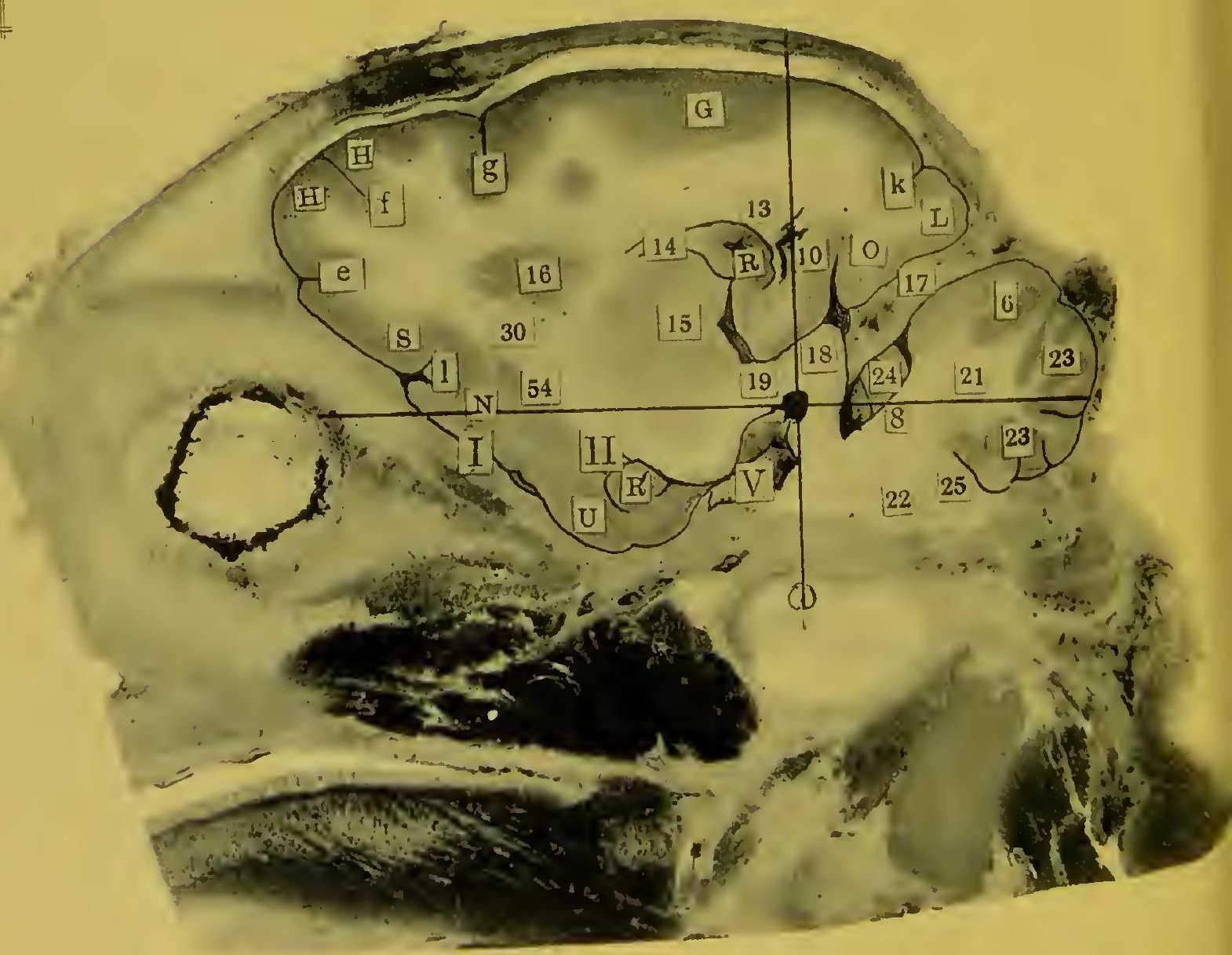

Lichtdruck von Albert Frisch, Berlin W:.

Fig. 2

Left. Sagittal Lamella 8. Cat 3.

Enlarged two diameters. Screen 3 

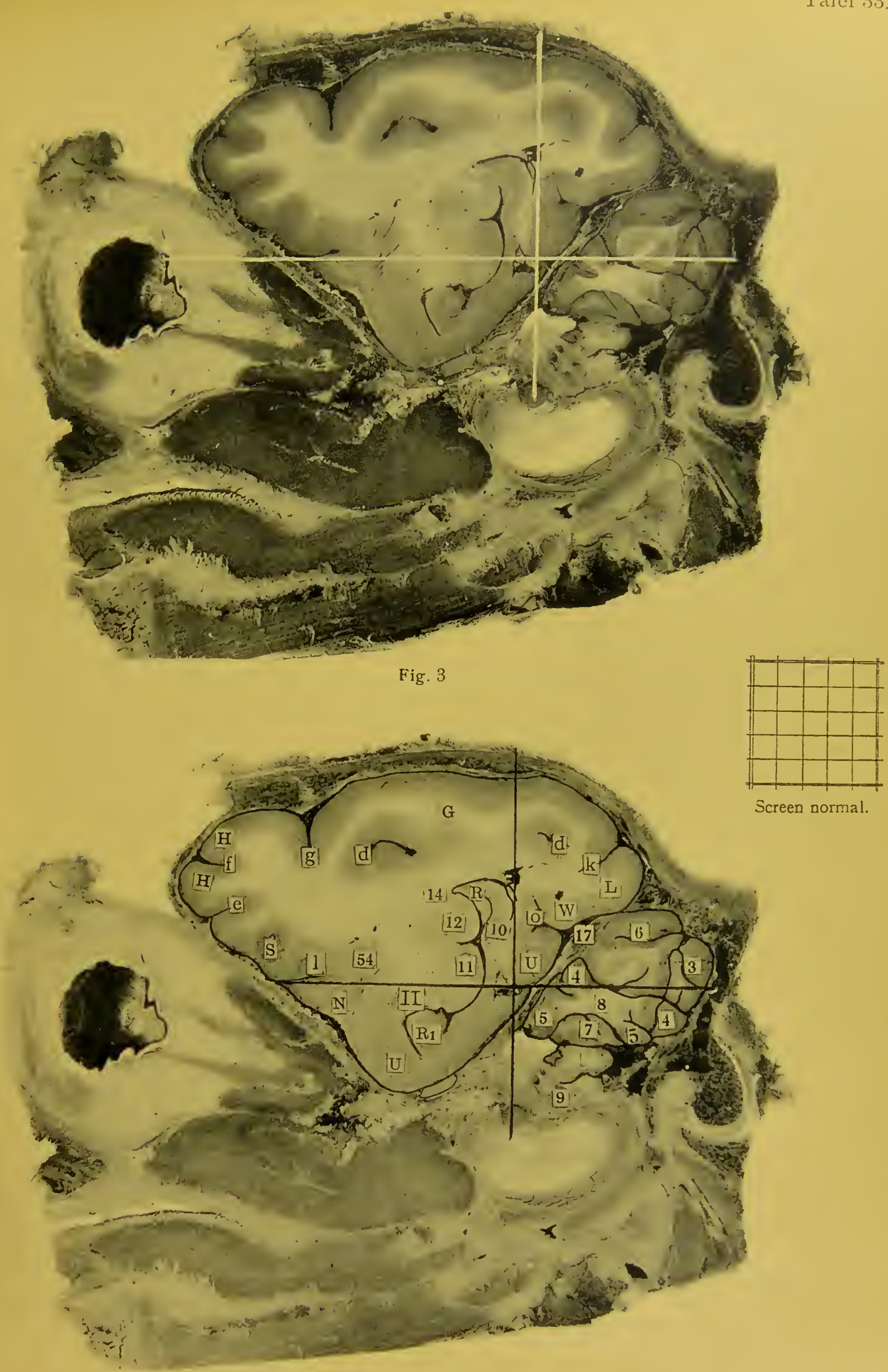

Fig. 4

Left. Sagittal Lamella 9. Cat 6. Enlarged two diameters. Screen normal. 

Journal f. Psychol. u. Neurol. XVIII. Ergänzungsheft.

(Clarke and Henderson, Brain of Cat.)

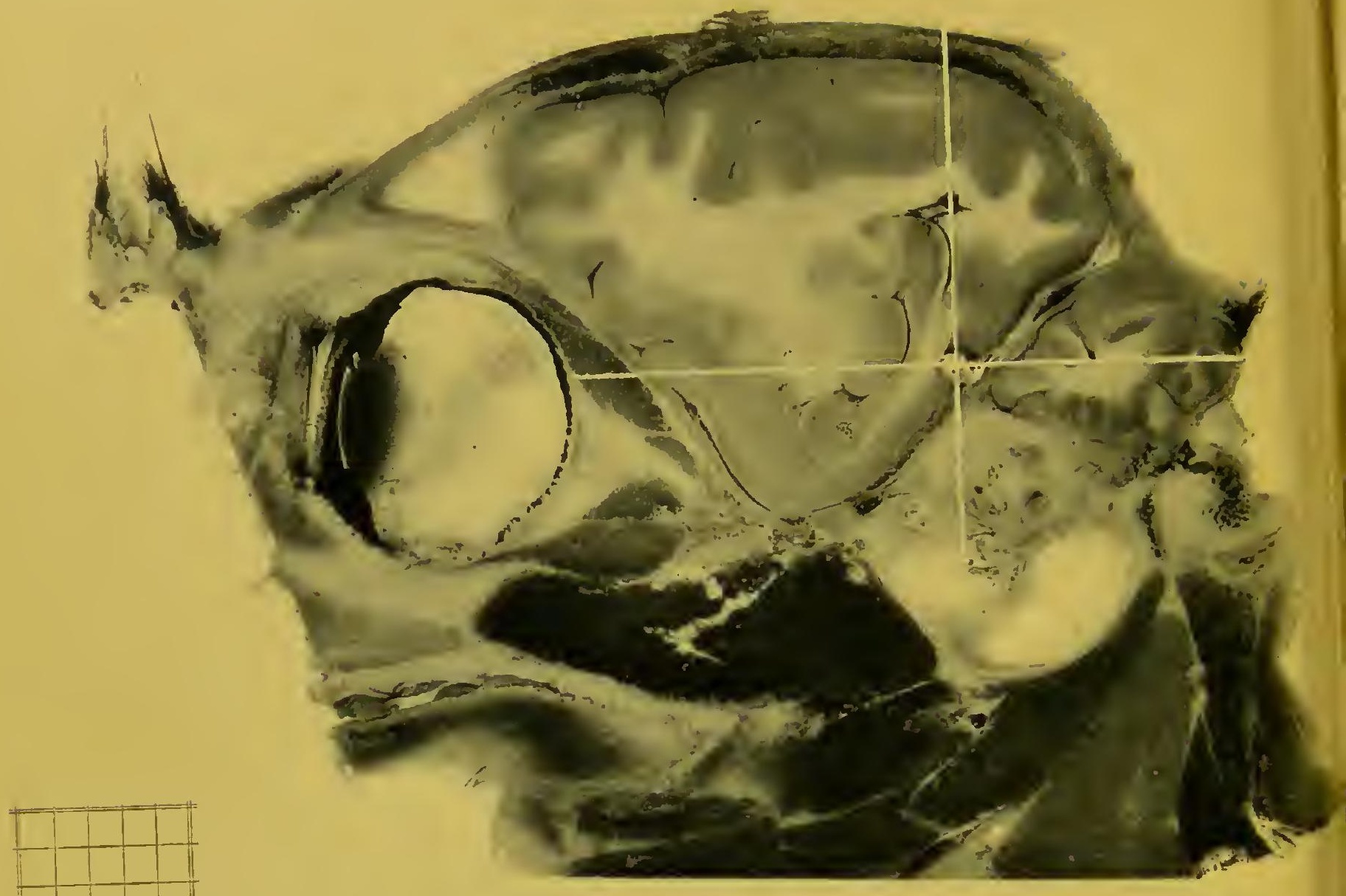

Fig. 1

Screen 3....

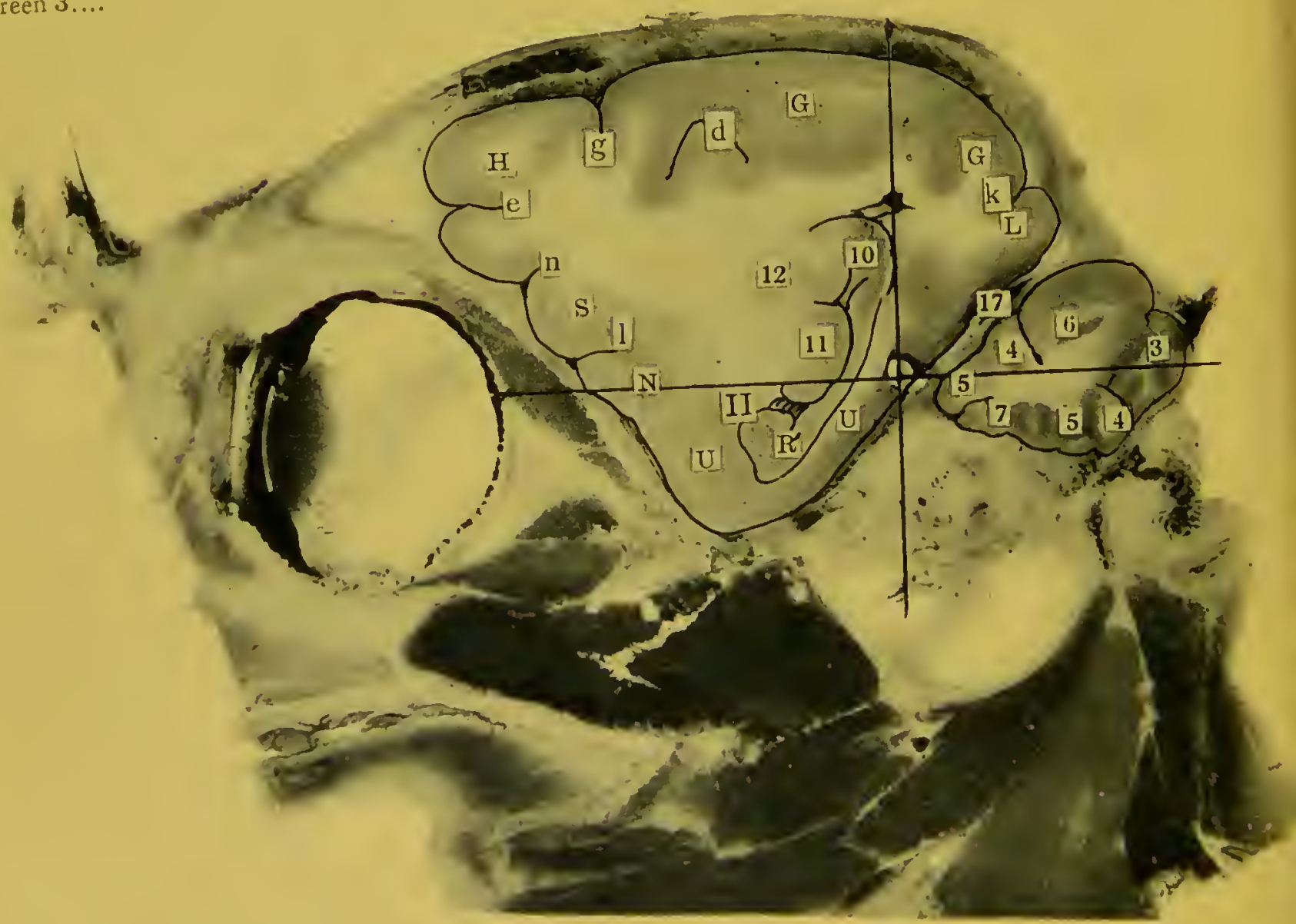

Fig.'2

Left. Sagittal Lamella 10. Cat 3. Enlarged two diameters. Screen 3. 

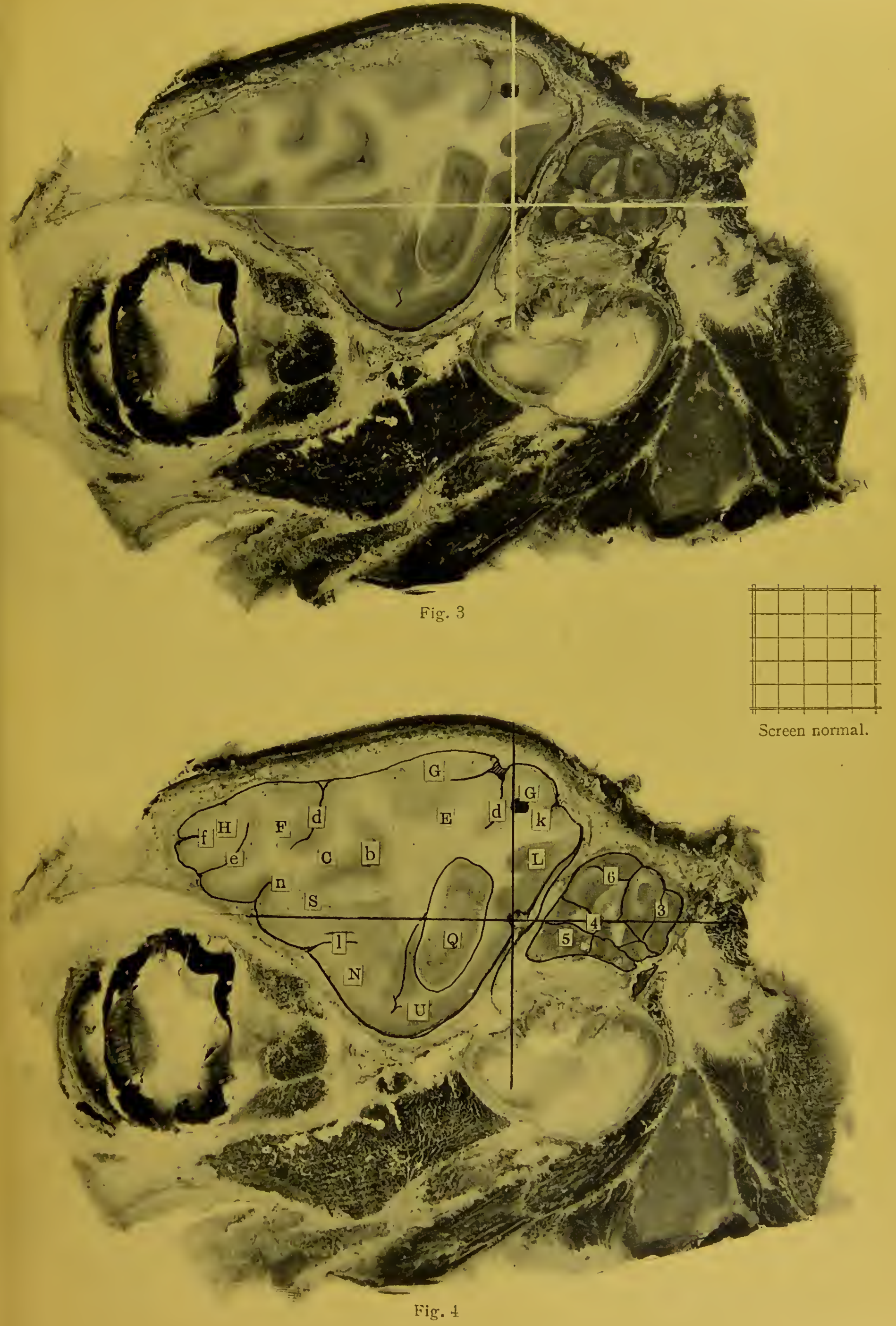

Left. Sagittal Lamella 11. Cat 1. Enlarged two diameters. Screen normal. 


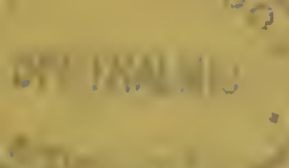



Journal f. Psychol. u. Neurol. XVIII. Ergänzungsheft.

(Clarke and Henderson, Brain of Cat.)

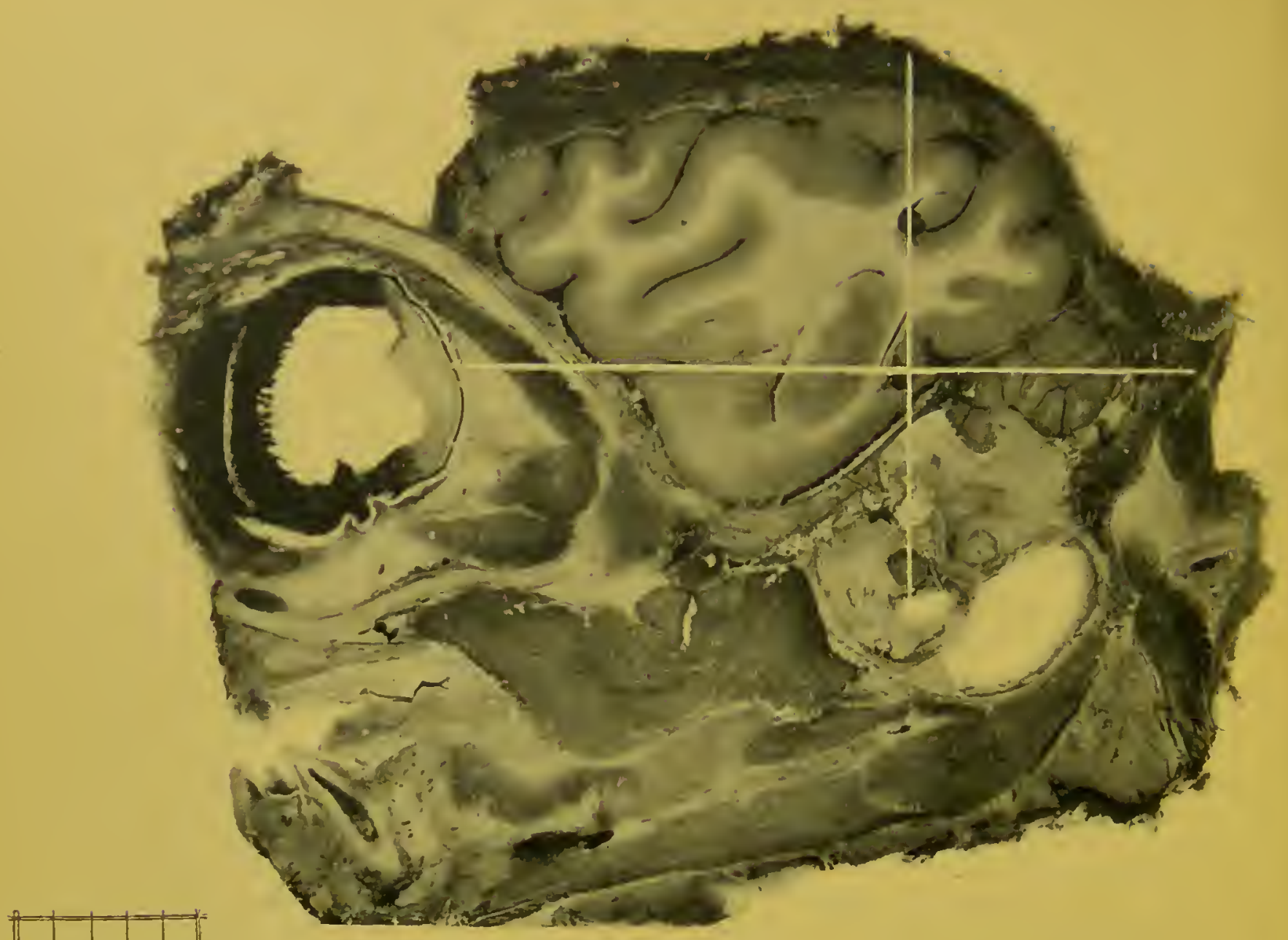

Fig. 1

Screen normal.

Lichtdruck von Albert Frisch, Berlin W.

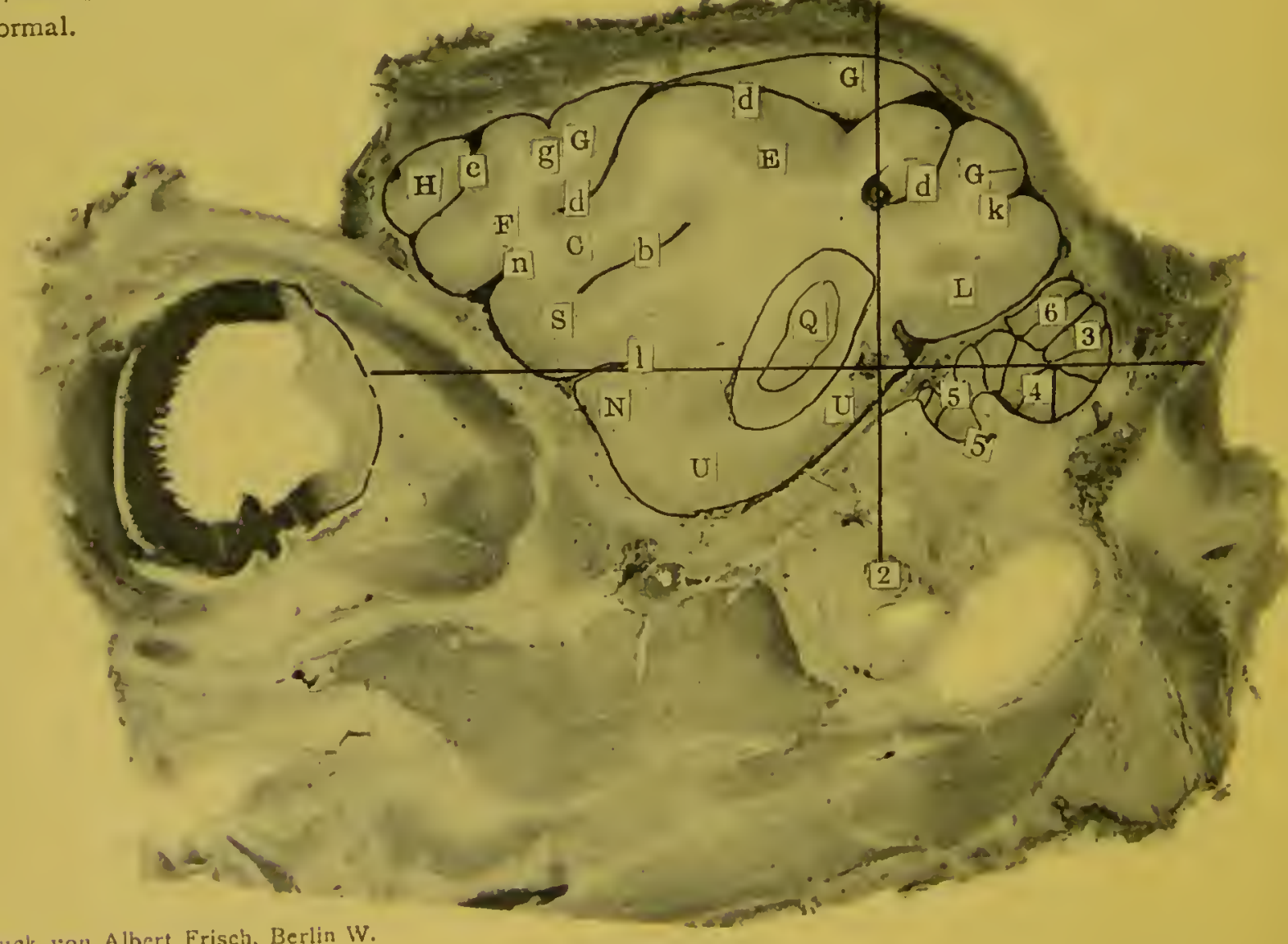

Fig. 2

Left. Sagittal Lamella 12. Cat 6.

Enlarged two diameters. Screen normal. 


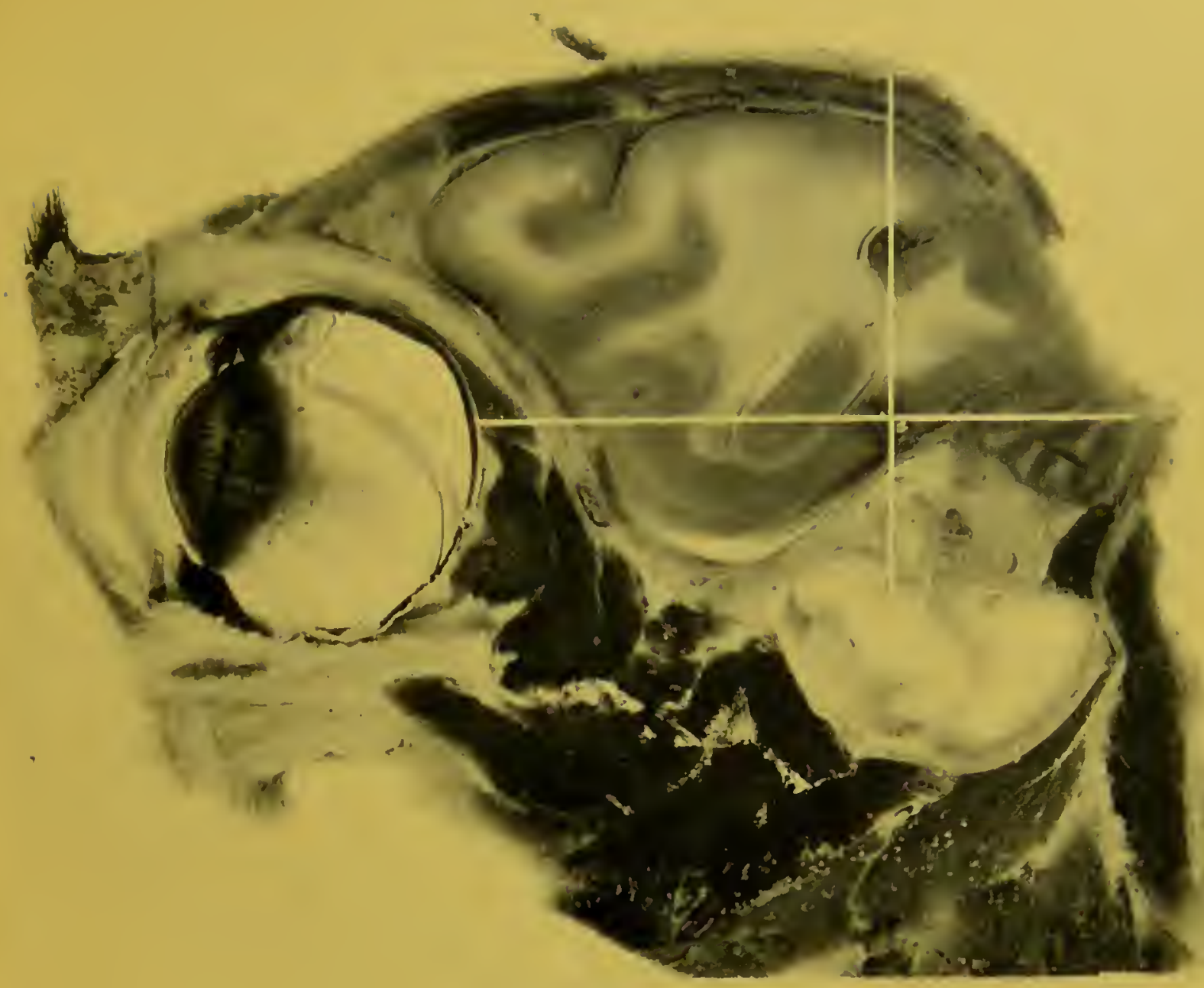

Fig. 3
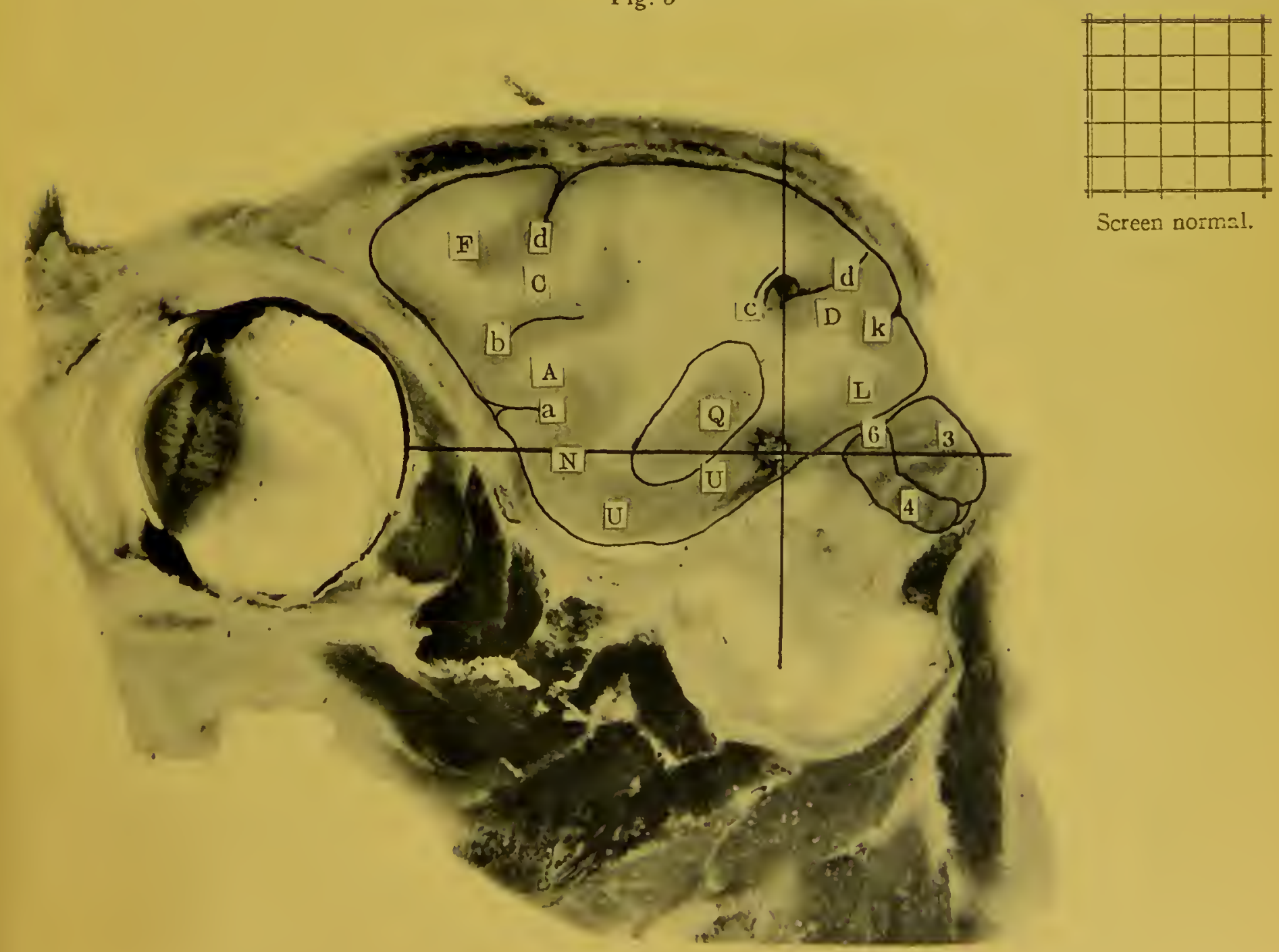

Fig. 4

Left. Sagittal Lamella 13. Cat 6.

Enlarged two diameters. Screen normal. 
Exmanto 

Journal f. Psychol. u. Neurol. XVIII. Ergänzungsheft.

(Clarke and Henderson, Brain of Cat.)


Fig. 1

Screen normal.

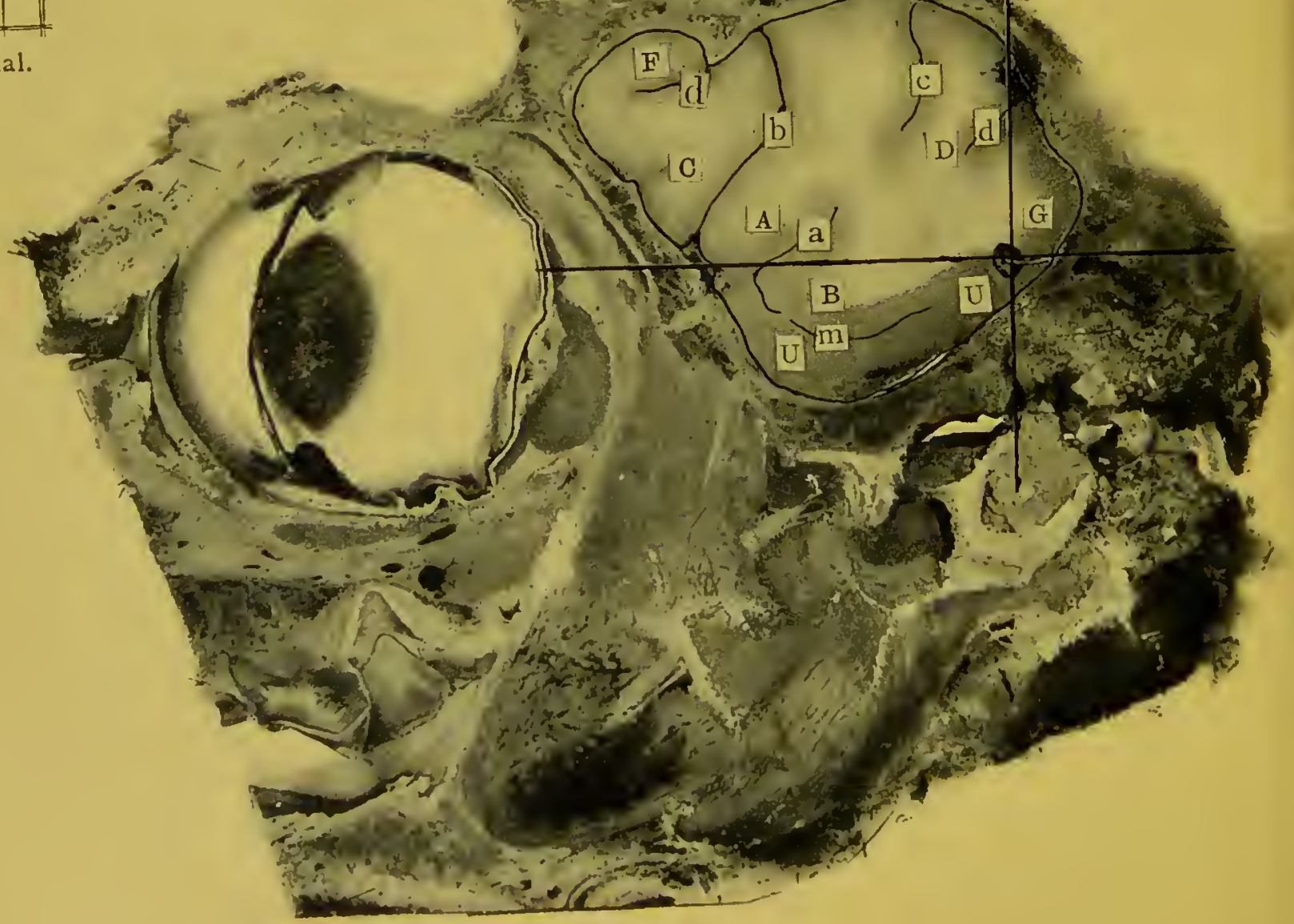

Fig. 3

Left. Sagittal Lamella 14. Cat 4.

Finlarged two diameters. Screen normal. 


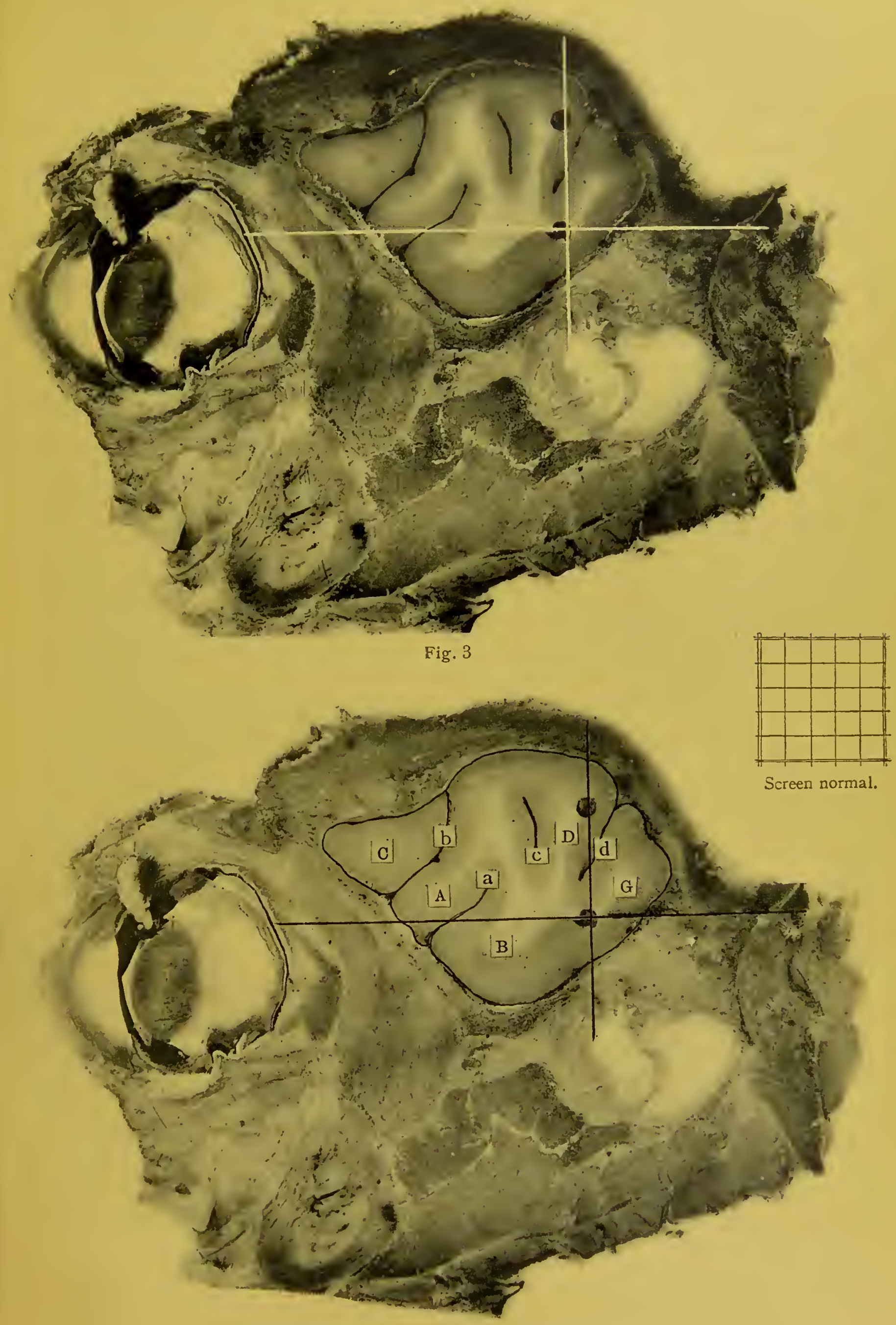

Fig. 4

Left. Sagittal Lamella 15. Cat 6. Eniarged two diameters. Screen normal. 
Hindalo 

Journal f. Psychol. u. Neurol. XVIII. Ergänzungsheft.

(CJarke and Henderson, Brain of Cat.)

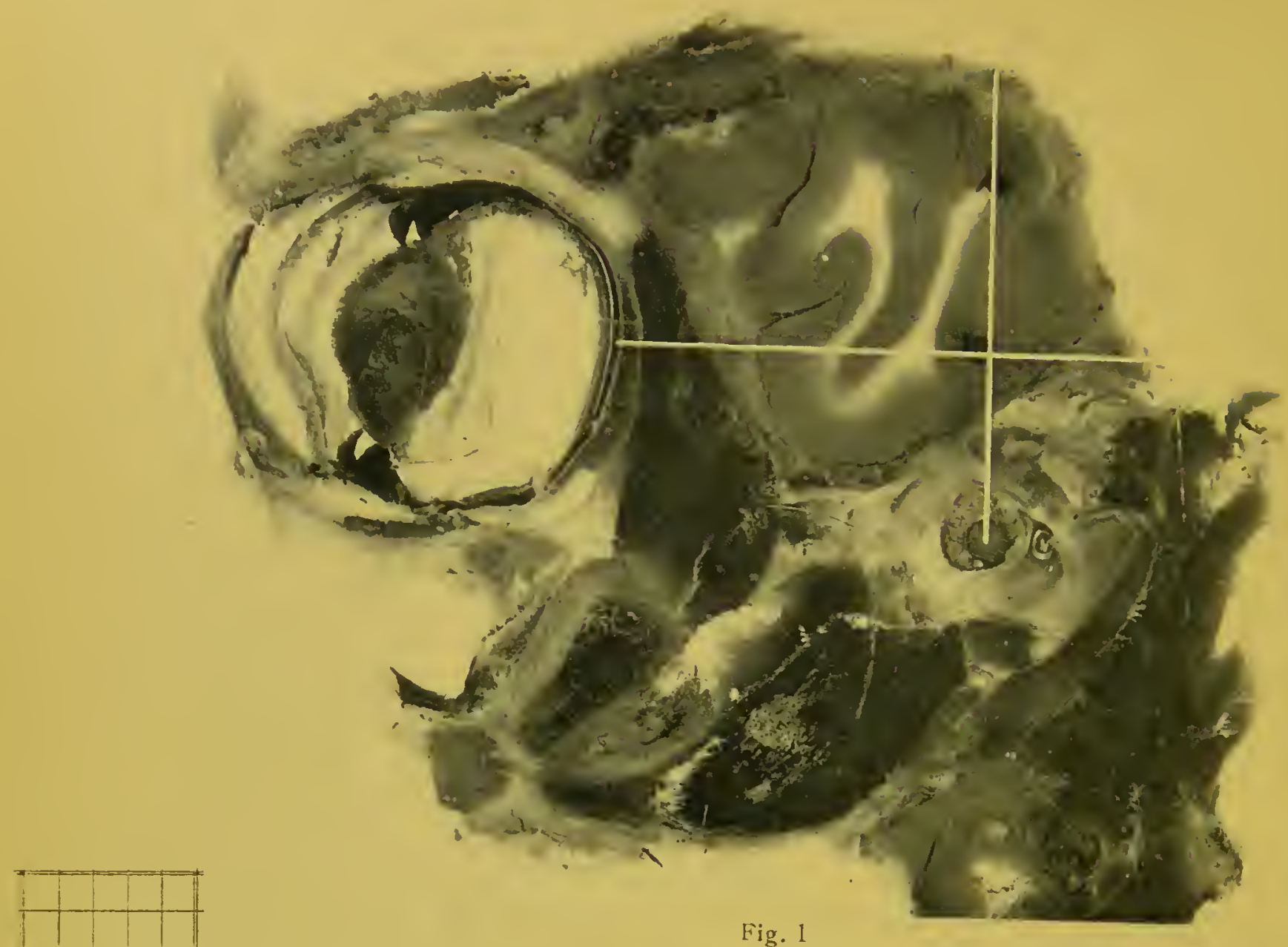

Fig. 1

Screen $3 \ldots$

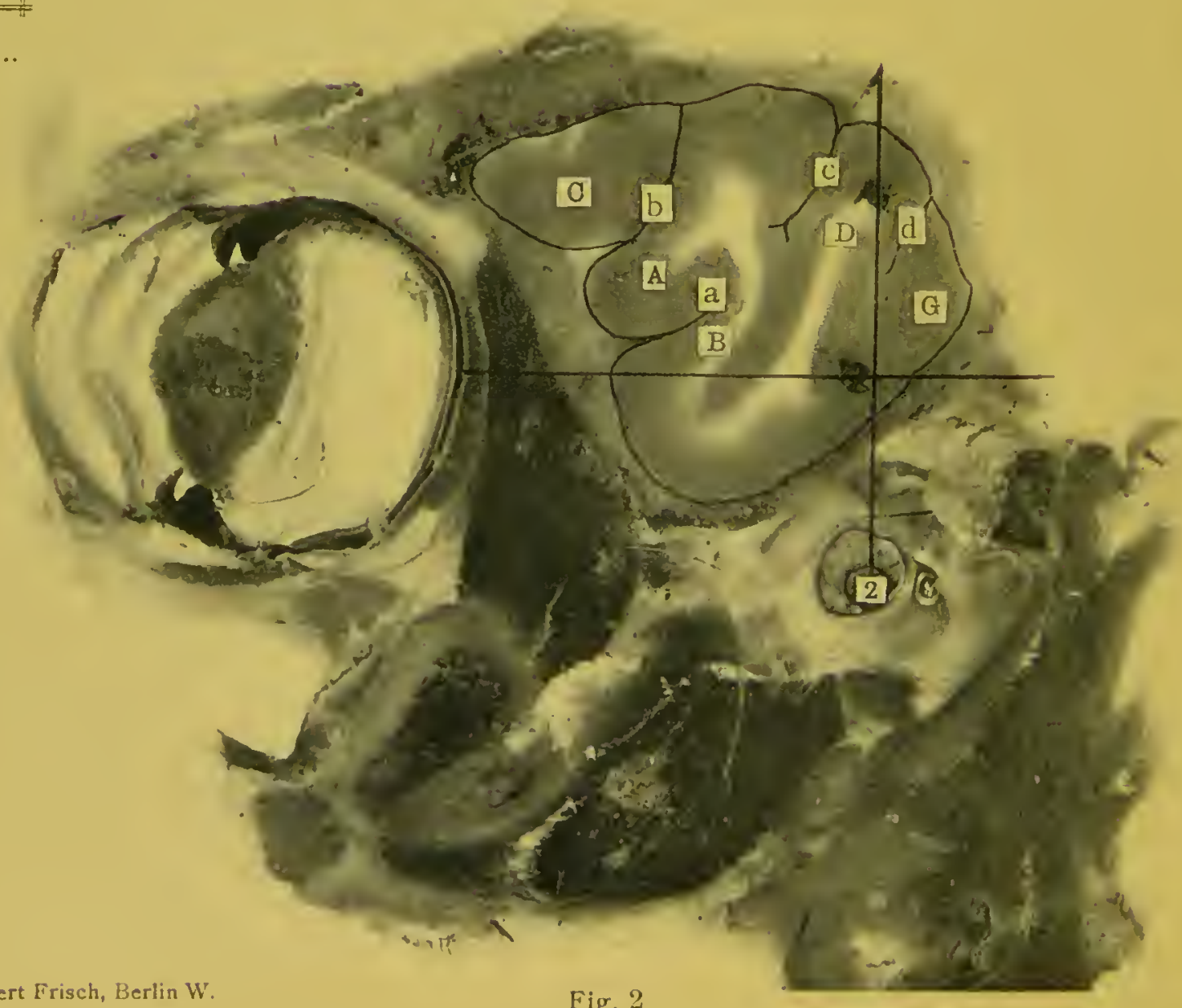

Left. Sagittal Lamella 16. Cat 3.

Enlarged two diameters. Screen 3. 



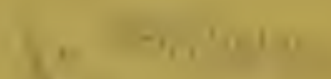

Yom $\therefore=$ 
Journal f. Psychol. u. Neurol. XVIII. Ergänzungsheft.

(Clarke and Henderson. Brain of Cat.)

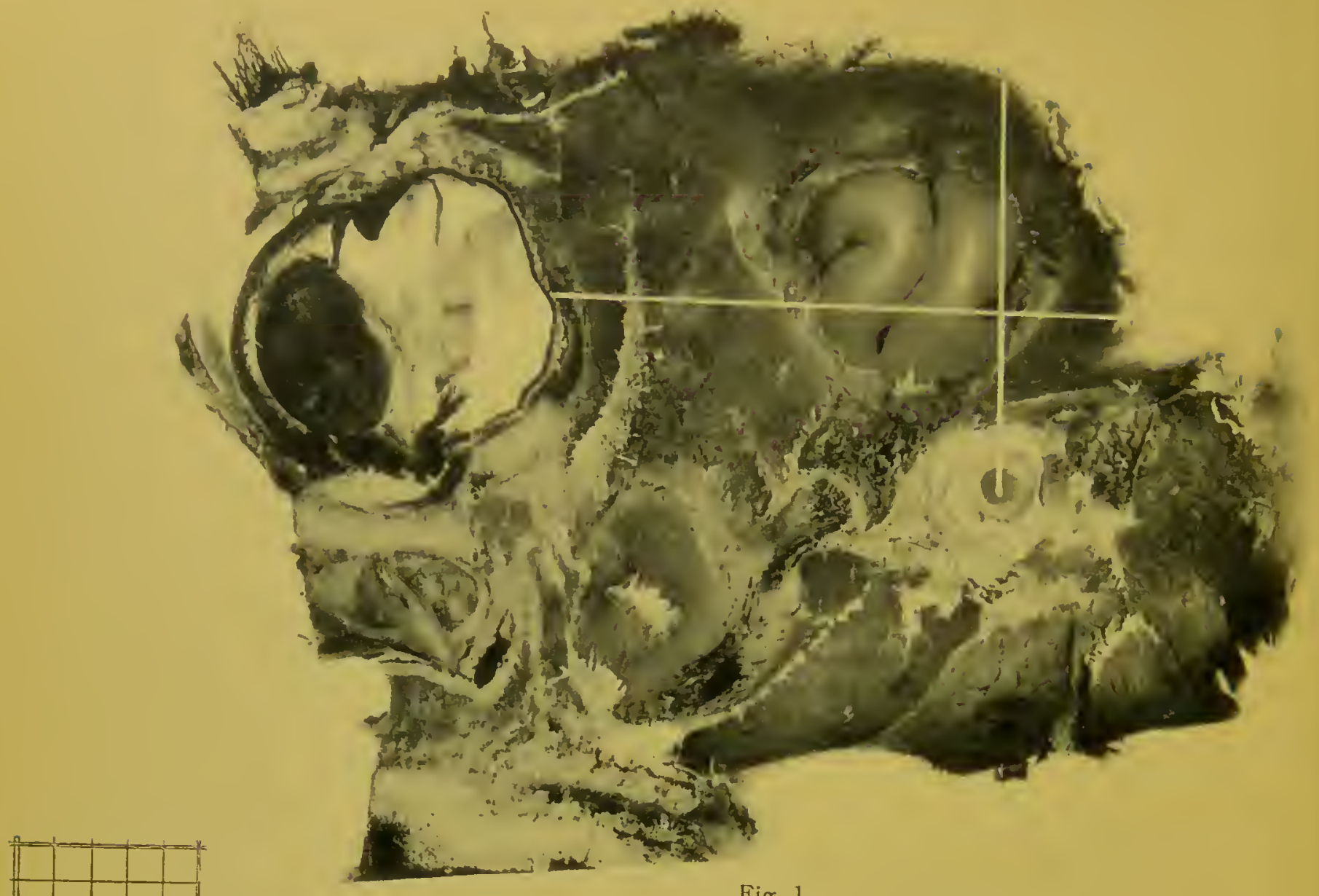

Fig. 1

Screen normal.

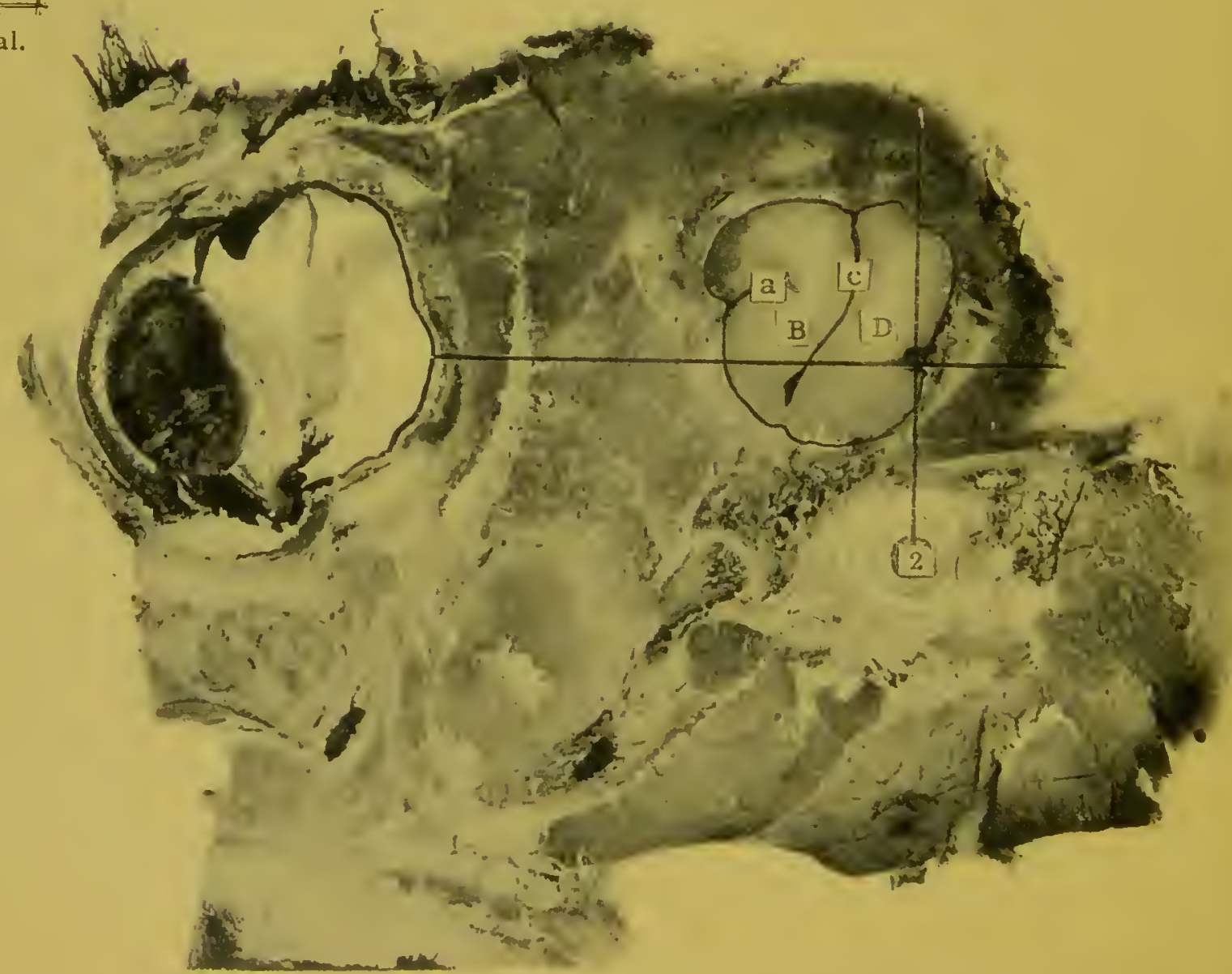

Fig. 2 


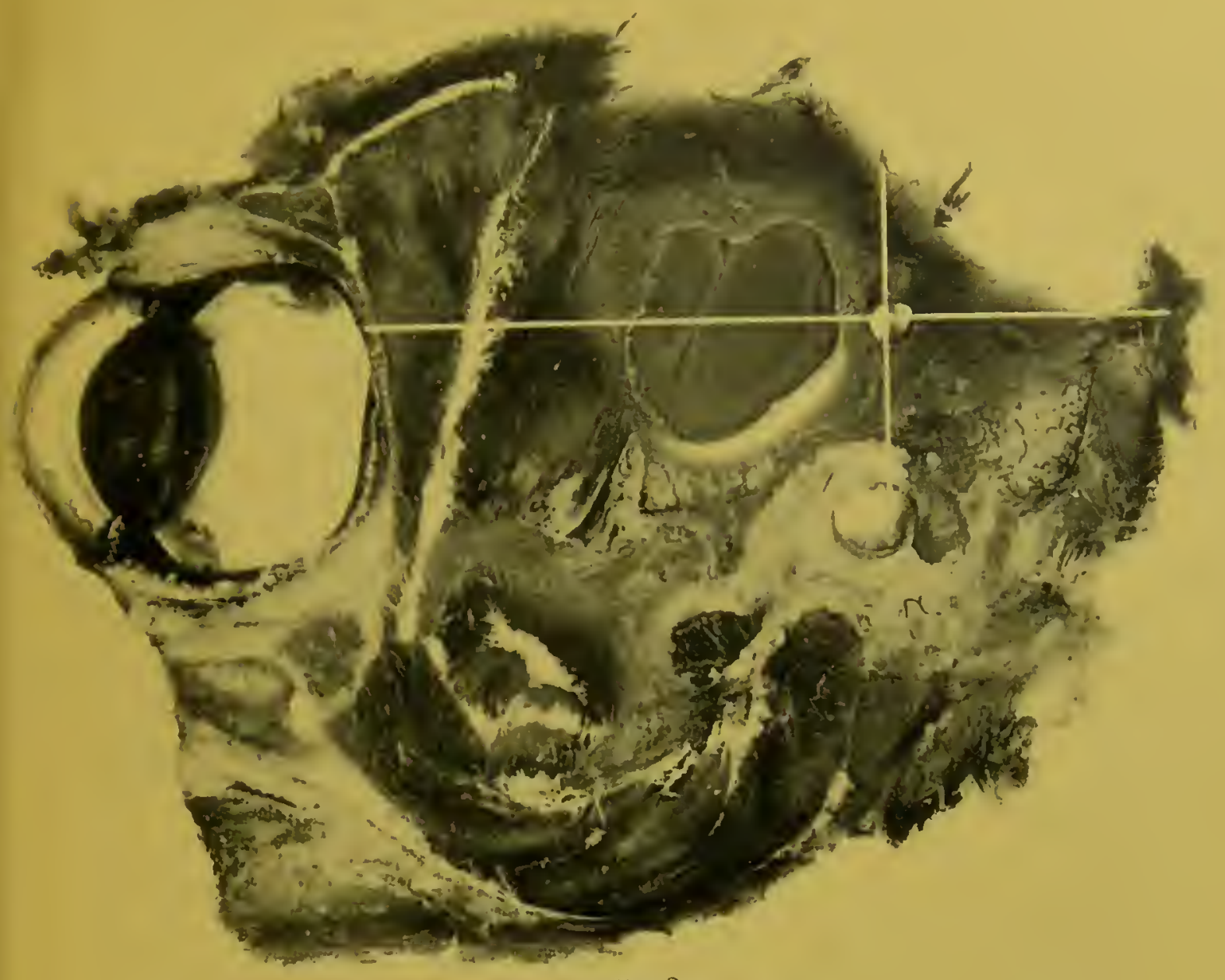

Fig. 3

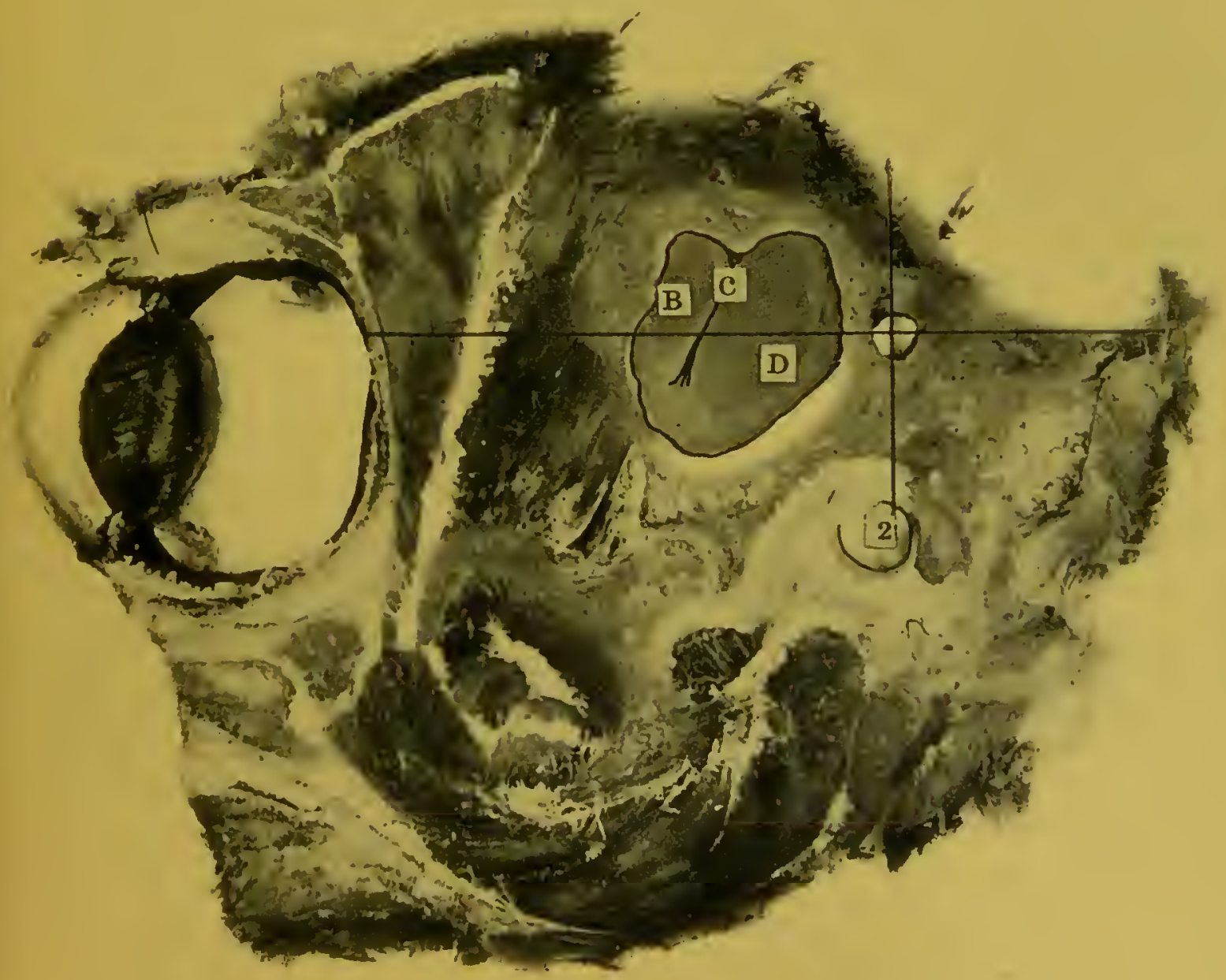

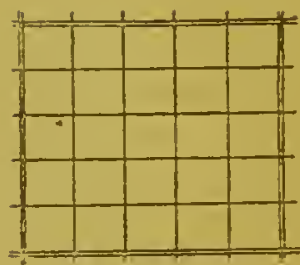

Screen 2....

Fig. 4

Left. Sagittal Lamella 19. Cat 2. Enlarged two diameters. Screen 2. 




